\title{
Harmonic sums and Mellin transforms up to two-loop order
}

\author{
Johannes Blümlein and Stefan Kurth \\ DESY-Zeuthen, Platanenallee 6, D-15735 Zeuthen, Germany
}

(Received 5 October 1998; published 14 June 1999)

\begin{abstract}
A systematic study is performed on the finite harmonic sums up to level four. These sums form the general basis for the Mellin transforms of all individual functions $f_{i}(x)$ of the momentum fraction $x$ emerging in the quantities of massless QED and QCD up to two-loop order, as the unpolarized and polarized splitting functions, coefficient functions, and hard scattering cross sections for space and timelike momentum transfer. The finite harmonic sums are calculated explicitly in the linear representation. Algebraic relations connecting these sums are derived to obtain representations based on a reduced set of basic functions. The Mellin transforms of all the corresponding Nielsen functions are calculated. [S0556-2821(99)04411-2]

PACS number(s): 11.15.Bt, 12.38.Bx
\end{abstract}

\section{INTRODUCTION}

For the study of the scaling violations of deep-inelastic scattering structure functions and other hard scattering processes different techniques were developed [1,2]. Because of mass factorization the processes can in general be described by a Mellin convolution of the parton densities and coefficient functions or the hard scattering cross sections in the c.m. subsystem, respectively. The Mellin convolution of two functions $A(x)$ and $B(x)$ is described by

$$
[A \otimes B](x)=\int_{0}^{1} d x_{1} \int_{0}^{1} d x_{2} \delta\left(x-x_{1} x_{2}\right) A\left(x_{1}\right) B\left(x_{2}\right)
$$

In most of the approaches the cross sections are expressed using the above relation. In the case of the evolution equations of the parton densities one has to solve coupled integrodifferential equations in $x$ space [1]. On the other hand one may consider the integral transformation [3]

$$
\mathbf{M}\left[f_{i}\right](N)=\int_{0}^{1} d x x^{N-1} f_{i}(x),
$$

for the functions $\left.f_{i}(x) \epsilon \mathcal{C}_{0}^{\infty}\right] 0,1\left[\right.$, cf. [4], for which $\mathbf{M}\left[f_{i}\right]$ exists. Here $N$ denotes the integer moment index.

Since

$$
\mathbf{M}[A \otimes B](N)=\mathbf{M}[A](N) \cdot \mathbf{M}[B](N),
$$

the convolutions are mapped into ordinary products leading to considerable simplifications in most of the problems [2]. The Mellin transforms $\mathbf{M}\left[f_{i}\right](N)$ appear as the genuine quantities in the operator product expansion at integer $N$. The quantities $\mathbf{M}[A](N)$ may be analytically continued to complex values of the argument. Complicated convolution relations, which emerge in some applications, can be handled easily by applying the transform Eq. (2), cf. [5]. Fast evolution codes operating at high numerical precision are based on this prescription [2]. Specific resummations are most easily implemented via the Mellin representation [6,7] allowing also for a thorough account of the commutation relations between the singlet matrices $[8,7]$, which is more difficult to be established in the $x$-space representation. The procedure can also be applied for analyses of the hadronic final states [9].

For the subsequent description it appears useful to enlarge the linear space $\left.\mathcal{C}_{0}^{\infty}\right] 0,1\left[\right.$ to $\left.\left.\left.\hat{\mathcal{C}}_{0}^{\infty}\right] 0,1\right]=\mathcal{C}_{0}^{\infty}\right] 0,1[\cup$ $\left\{\theta(1-x), \delta^{(k)}(1-x), k \epsilon \mathbf{N}\right\}$. Here $\theta(1-x)$ and $\delta(1-x)$ denote the Heaviside function and $\delta$-distribution [11], respectively. The Mellin convolution $\otimes$, Eq. (1), induces a multiplication in $\left.\left.\hat{\mathcal{C}}_{0}^{\infty}\right] 0,1\right] .{ }^{1}$ This operation is mapped onto ordinary multiplication in the linear space of the Mellin transforms.

The functions $f_{i}(x)$ emerging in perturbative calculations in massless quantum field theories belong to the class discussed by Nielsen [12-14] and their Mellin convolutions. By explicit calculation we will show that the Mellin transforms of these functions can be represented by linear combinations of the finite harmonic sums

$$
\begin{aligned}
& S_{k_{1}, \ldots, k_{m}}(N)= \sum_{n_{1}=1}^{N} \frac{\left[\operatorname{sign}\left(k_{1}\right)\right]^{n_{1}}}{n_{1}^{\left|k_{1}\right|}} \sum_{n_{2}=1}^{n_{1}} \frac{\left[\operatorname{sign}\left(k_{2}\right)\right]^{n_{2}}}{n_{2}^{\left|k_{2}\right|}} \ldots \\
& \times \sum_{n_{m}=1}^{n_{m-1} \frac{\left[\operatorname{sign}\left(k_{m}\right)\right]^{n_{m}}}{n_{m}^{\left|k_{m}\right|}},} \\
& N \epsilon \mathbf{N}, \quad \forall l, k_{l} \neq 0 .
\end{aligned}
$$

Up to the two-loop level harmonic sums up to level $\lambda$ $=\sum_{j=1}^{m}\left|k_{j}\right|=4$ contribute. The finite harmonic sums are related to the multiple $\zeta$ values $^{2}[15-17] \sigma_{k_{1}}, \ldots, k_{m}, k_{1} \neq 1$ in the limit $N \rightarrow \infty$.

\footnotetext{
${ }^{1}$ For similar considerations concerning other integral transforms, see e.g. [10].

${ }^{2}$ The notion derives from the case of single-parameter sums $\sigma_{k}$ $=\sum_{n=1}^{\infty} 1 / n^{|k|} \equiv \zeta(k)$ for $k>1$ and $\zeta$ denoting the Riemann $\zeta$ function. Most applications discuss positive indices $k_{i}$ and refer to a somewhat different ordering in nesting the sums by demanding $n_{i-1}>n_{i}$ which, however, is linearly related to the treatment in the present paper. Because alternating sums do emerge in most of the applications in quantum field theories we refer to this generalized case subsequently.
} 
Besides the linear representations various algebraic relations connect the different harmonic sums. A still higher symmetry is obeyed by the multiple $\zeta$ values $[18,19]$. A comparison of the linear representations with those induced by the algebraic relations may yield rather non-trivial relations between Nielsen functions [20]. The systematic classification of the finite harmonic sums appears to be carried out in a somewhat easier way than the Mellin transforms of the various Nielsen functions themselves which is due to the possibility to choose different arguments and linear combinations of convolutions leading to equivalent representations in the latter case. Also the algebraic structures are more easily recognized for the harmonic sums.

It is the aim of the present paper to derive the Mellin transforms of all individual functions $f_{i}(x)$ emerging in the description of the splitting and coefficient functions in massless QED and QCD up to two-loop order. This also includes the coefficient functions for time-like processes, such as fragmentation functions, the Drell-Yan process and similar reactions. We investigate their relations to the finite harmonic sums and derive algebraic relations among them. They allow for a compact representation of all Mellin transforms over a fairly reduced set of basic functions.

The paper is organized as follows. In Sec. II we introduce the basic notations. The structure of anomalous dimensions and coefficient functions up to two-loop order in terms of Nielsen functions is discussed Sec. III, where we consider the Wilson coefficients of $F_{L}\left(x, Q^{2}\right)$ and the coefficient function $c_{2}^{\mathrm{NS}}\left(x, Q^{2}\right)$ as examples. Section IV contains the explicit linear representations of all harmonic sums up to level four. Section V deals with algebraic relations between the finite harmonic sums and Sec. VI contains the conclusions. The individual Mellin transforms for the functions $f_{i}(x)$ emerging in the different splitting and coefficient functions are given in the Appendix.

\section{HARMONIC SUMS AND MELLIN TRANSFORMS IN FIELD THEORY}

The coefficient [21-25] and splitting functions [26-29], can be expressed as linear combinations of products of the Nielsen functions [12-14]

$$
\mathrm{S}_{n, p}(x)=\frac{(-1)^{n+p-1}}{(n-1) ! p !} \int_{0}^{1} \frac{d z}{z} \log ^{n-1}(z) \log ^{p}(1-z x) .
$$

We introduce the notion of the degree of these transcendental functions as their transcendentality $p+n$. The polylogarithms are given by

$$
\operatorname{Li}_{n}(x)=\frac{d \mathrm{Li}_{n+1}(x)}{d \log (x)}=\mathrm{S}_{n-1,1}(x) .
$$

The logarithms $\log (1 \pm x)$ are related to the dilogarithm by [30]

$$
\frac{d \mathrm{Li}_{2}( \pm x)}{d \log (x)}=\mathrm{Li}_{1}( \pm x)=-\log (1 \mp x)
$$

and

$$
\operatorname{Li}_{0}(x)=\frac{x}{1-x}
$$

Similarly,

$$
\frac{d \mathrm{~S}_{n, p}(x)}{d \log (x)}=\mathrm{S}_{n-1, p}(x)
$$

holds. While the $\log$ arithms $\log (1 \pm x)$ and $\log (x)$ are of transcendentality 1 , the polylogarithm $\mathrm{Li}_{n}(x)$ has transcendentality $n$. The transcendentality of the measures

$$
\frac{d x}{1 \pm x} \text { and } \frac{d x}{x}
$$

is defined to be 1 . For general one-dimensional integrals over the product of a number of Nielsen functions and their derivatives the transcendentality determines itself as the sum of the transcendentalities of the factors of the integrand and the measure.

The simplest harmonic sums $S_{ \pm 1}(N)^{3}$ are related to the Mellin transforms

$$
\begin{aligned}
S_{1}(N) & =\int_{0}^{1} d x \frac{x^{N}-1}{x-1} \equiv \mathbf{M}\left[\left(\frac{1}{x-1}\right)_{+}\right](N) \\
S_{-1}(N) & =\int_{0}^{1} d x \frac{(-x)^{N}-1}{x+1} \\
& \equiv(-1)^{N} \mathbf{M}\left[\left(\frac{1}{x+1}\right)\right](N)-\log (2) .
\end{aligned}
$$

Here the + -prescription is defined by

$$
\int_{0}^{1} d x g(x)[f(x)]_{+}=\int_{0}^{1} d x[g(x)-g(1)] f(x) .
$$

The general single harmonic sums $S_{ \pm k}(N), k>0$ are obtained by the iterated integrals

$$
\begin{aligned}
S_{k}(N) & =\int_{0}^{1} \frac{d x_{1}}{x_{1}} \int_{0}^{x_{1}} \frac{d x_{2}}{x_{2}} \ldots \int_{0}^{x_{k-1}} \frac{x_{k}^{N}-1}{x_{k}-1} \\
& =\frac{(-1)^{k-1}}{(k-1) !} \int_{0}^{1} d x \log ^{k-1}(x) \frac{x^{N}-1}{x-1} \\
S_{-k}(N) & =\int_{0}^{1} \frac{d x_{1}}{x_{1}} \int_{0}^{x_{1}} \frac{d x_{2}}{x_{2}} \ldots \int_{0}^{x_{k-1}} \frac{\left(-x_{k}\right)^{N}-1}{x_{k}+1} \\
& =\frac{(-1)^{k-1}}{(k-1) !} \int_{0}^{1} d x \log ^{k-1}(x) \frac{(-x)^{N}-1}{x+1} .
\end{aligned}
$$

\footnotetext{
${ }^{3} S_{1}(N)$ occurs as the only function being not a polynomial in the 1-loop anomalous dimensions $\gamma_{q q}^{(0)}$ and $\gamma_{g g}^{(0)}$ [26].
} 
Further alternating or non-alternating summation does naturally lead to Mellin transforms of Nielsen functions or their products of higher transcendentality. The integral representation of a general harmonic sum $S_{k_{l}, \ldots, k_{1}}(N)$ can be obtained recursively from Eqs. (14), (15) using

$$
\begin{gathered}
\sum_{k=1}^{n} \frac{x^{k}}{k^{l}}=\frac{(-1)^{l-1}}{(l-1) !} \int_{0}^{x} d z \log ^{l-1}(z) \frac{z^{n}-1}{z-1} \\
\sum_{k=1}^{n} \frac{(-z)^{k}}{k^{l}}=\frac{(-1)^{l-1}}{(l-1) !} \int_{0}^{x} d z \log ^{l-1}(z) \frac{(-z)^{n}-1}{z+1} .
\end{gathered}
$$

In the limit $N \rightarrow \infty$ the harmonic sums yield

$$
\tilde{\sigma}_{k_{l}, \ldots, k_{1}}=\lim _{N \rightarrow \infty} S_{k_{l}, \ldots, k_{1}}(N)
$$

with $\tilde{\sigma}_{k_{l}, \ldots, k_{1}}$ being a polynomial of multiple $\zeta$ values $\sigma_{k_{m} \ldots k_{1}}, k_{m} \neq 1$, and $\sigma_{+1}$,

$$
\sigma_{+1}=\int_{0}^{1} d x \frac{1}{1-x}
$$

As already the case for the representation of $S_{-1}(N)$, Eq. (12), specific numbers as $\log (2)$ emerge, which multiply harmonic sums of lower level or Mellin transforms of functions of lower transcendentality. Although it is not known yet whether all these constants are transcendental numbers we apply the notion of transcendentality introduced above also to them to obtain a uniform description. Up to transcendentality 4 only a single constant per level emerges: $\log (2), \zeta(2), \zeta(3), \mathrm{Li}_{4}(1 / 2)$. At higher levels more constants contribute. For convenience we follow the choice of Refs. [31-35]. The constants are at level $5 \zeta(5)$ and $\operatorname{Li}_{5}(1 / 2)$, at level $6 \operatorname{Li}_{6}(1 / 2)$ and $\sigma_{-5,-1}$, and at level 7 $\operatorname{Li}_{7}(1 / 2), \sigma_{-5,-1,-1}$ and $\sigma_{5,-1,-1}$, which are believed to be not further reducible in terms of $\zeta$ values apart form reordering. ${ }^{4}$

\section{COEFFICIENT FUNCTIONS AT $O\left(\alpha_{s}^{2}\right)$}

The Mellin transforms of the splitting functions up to next-to-leading order [28] are known for a long time. Most of the contributing functions can be reduced to polynomials in $S_{ \pm k}(N)$ except $\mathrm{Li}_{2}(x) /(1+x)$ which is related to the sum $S_{-2,1}(N)$, cf. Sec. IV. ${ }^{5}$ Some functions $f_{i}(x)$ emerge in a combination leading to a simpler Mellin transform. This is the case for $\mathrm{Li}_{2}(-x)+\log (x) \log (1+x)$ which can be expressed by

\footnotetext{
${ }^{4}$ For recent reviews on multiple $\zeta$ values see [36].

${ }^{5}$ See also Ref. [37].
}

$$
\mathrm{Li}_{2}(-x)+\ln x \ln (1+x)=-\frac{1}{2} \widetilde{\Phi}(x)+\frac{1}{4} \ln ^{2} x-\frac{\zeta(2)}{2}
$$

The Mellin transform of the function

$$
\widetilde{\Phi}(x)=\int_{x /(1+x)}^{1 /(1+x)} \frac{d z}{z} \ln \left(\frac{1-z}{z}\right)
$$

itself turns out to be more compact than that of $\mathrm{Li}_{2}(-x)$ and $\log (x) \log (1+x)$ individually.

The Mellin transforms of the two-loop coefficient functions are more complicated than those of the splitting functions. The simplest among them are the coefficient functions of the longitudinal structure function $F_{L}\left(x, Q^{2}\right)$, which is given by

$$
\begin{aligned}
F_{L}\left(x, Q^{2}\right)= & x\left\{C_{\mathrm{NS}}\left(x, Q^{2}\right) \otimes f_{\mathrm{NS}}\left(x, Q^{2}\right)+\delta_{f}\left[C_{\mathrm{S}}\left(x, Q^{2}\right)\right.\right. \\
& \left.\left.\otimes \Sigma\left(x, Q^{2}\right)+C_{\mathrm{g}}\left(x, Q^{2}\right) \otimes G\left(x, Q^{2}\right)\right]\right\}
\end{aligned}
$$

in the case of pure photon exchange. The combinations of parton densities are

$$
\begin{aligned}
f_{\mathrm{NS}}\left(x, Q^{2}\right) & =\sum_{i=1}^{N_{f}} e_{i}^{2}\left[q_{i}\left(x, Q^{2}\right)+\bar{q}_{i}\left(x, Q^{2}\right)\right], \\
\Sigma\left(x, Q^{2}\right) & =\sum_{i=1}^{N_{f}}\left[q_{i}\left(x, Q^{2}\right)+\bar{q}_{i}\left(x, Q^{2}\right)\right] .
\end{aligned}
$$

$G\left(x, Q^{2}\right)$ denotes the gluon density, $e_{i}$ the electric charge, and $\delta_{f}=\left(\sum_{i=1}^{N_{f}} e_{i}^{2}\right) / N_{f}$, with $N_{f}$ the number of active flavors.

The coefficient functions $C_{i}\left(x, Q^{2}\right)$ read

$$
\begin{gathered}
C_{\mathrm{NS}}\left(z, Q^{2}\right)=a_{s} c_{L, q}^{(1)}(z)+a_{s}^{2} c_{L, q}^{(2), N S}(z) \\
C_{\mathrm{S}}\left(z, Q^{2}\right)=a_{s}^{2} c_{L, q}^{(2), P S}(z) \\
C_{\mathrm{g}}\left(z, Q^{2}\right)=a_{s} c_{L, g}^{(1)}(z)+a_{s}^{2} c_{L, g}^{(2)}(z),
\end{gathered}
$$

where $a_{s}=\alpha_{s}\left(Q^{2}\right) /(4 \pi)$. The leading order coefficient functions are given by [38]

$$
\begin{aligned}
& c_{L, q}^{(1)}(z)=4 C_{F} z \\
& c_{L, g}^{(1)}(z)=8 N_{f} z(1-z) .
\end{aligned}
$$

In the modified minimal subtraction $\overline{(\mathrm{MS})}$ scheme the next leading order (NLO) coefficient functions read $[21,22]^{6}$

\footnotetext{
${ }^{6}$ Previous calculations $[39,40]$ turned out to be partly incorrect, whereas agreement was shown between Refs. [21,22] and [41]. The Refs. [40] were later corrected in Ref. [42].
} 


$$
\begin{aligned}
& c_{L, q}^{(2), N S}(z)=4 C_{F}\left(C_{A}-2 C_{F}\right) z\left\{4 \frac{6-3 z+47 z^{2}-9 z^{3}}{15 z^{2}} \ln z-4 \mathrm{Li}_{2}(-z)[\ln z-2 \ln (1+z)]\right. \\
& -8 \zeta(3)-2 \ln ^{2} z[\ln (1+z)+\ln (1-z)]+4 \ln z \ln ^{2}(1+z)-4 \ln z \operatorname{Li}_{2}(z)+\frac{2}{5}\left(5-3 z^{2}\right) \ln ^{2} z \\
& -4 \frac{2+10 z^{2}+5 z^{3}-3 z^{5}}{5 z^{3}}\left[\operatorname{Li}_{2}(-z)+\ln z \ln (1+z)\right]+4 \zeta(2)\left[\ln (1+z)+\ln (1-z)-\frac{5-3 z^{2}}{5}\right] \\
& \left.+8 \mathrm{~S}_{1,2}(-z)+4 \mathrm{Li}_{3}(z)+4 \mathrm{Li}_{3}(-z)-\frac{23}{3} \ln (1-z)-\frac{144+294 z-1729 z^{2}+216 z^{3}}{90 z^{2}}\right\} \\
& +8 C_{F}^{2} z\left\{\operatorname{Li}_{2}(z)+\ln ^{2} z-2 \ln z \ln (1-z)+\ln ^{2}(1-z)-3 \zeta(2)-\frac{3-22 z}{3 z} \ln z+\frac{6-25 z}{6 z} \ln (1-z)-\frac{78-355 z}{36 z}\right\} \\
& -\frac{8}{3} C_{F} N_{f} z\left\{2 \ln z-\ln (1-z)-\frac{6-25 z}{6 z}\right\} \\
& c_{L, q}^{(2), P S}(z)=\frac{16}{9 z} C_{F} N_{f}\left\{3\left(1-2 z-2 z^{2}\right)(1-z) \ln (1-z)+9 z^{2}\left[\mathrm{Li}_{2}(z)+\ln ^{2} z-\zeta(2)\right]\right. \\
& \left.+9 z\left(1-z-2 z^{2}\right) \ln z-9 z^{2}(1-z)-(1-z)^{3}\right\} \\
& c_{L, g}^{(2)}(z)=C_{F} N_{f}\left\{16 z\left[\operatorname{Li}_{2}(1-z)+\ln z \ln (1-z)\right]+\left(-\frac{32}{3} z+\frac{64}{5} z^{3}+\frac{32}{15 z^{2}}\right)\left[\operatorname{Li}_{2}(-z)+\ln z \ln (1+z)\right]\right. \\
& +\left(8+24 z-32 z^{2}\right) \ln (1-z)-\left(\frac{32}{3} z+\frac{32}{5} z^{3}\right) \ln ^{2} z+\frac{1}{15}\left(-104-624 z+288 z^{2}-\frac{32}{z}\right) \ln z \\
& \left.+\left(-\frac{32}{3} z+\frac{64}{5} z^{3}\right) \zeta(2)-\frac{128}{15}-\frac{304}{5} z+\frac{336}{5} z^{2}+\frac{32}{15 z}\right\}+C_{A} N_{f}\left\{-64 \operatorname{Li}_{2}(1-z)+\left(32 z+32 z^{2}\right)\right. \\
& \times\left[\operatorname{Li}_{2}(-z)+\ln z \ln (1+z)\right]+\left(16 z-16 z^{2}\right) \ln ^{2}(1-z)+\left(-96 z+32 z^{2}\right) \ln z \ln (1-z) \\
& +\left(-16-144 z+\frac{464}{3} z^{2}+\frac{16}{3 z}\right) \ln (1-z)+48 z \ln ^{2} z+\left(16+128 z-208 z^{2}\right) \ln z+32 z^{2} \zeta(2) \\
& \left.+\frac{16}{3}+\frac{272}{3} z-\frac{848}{9} z^{2}-\frac{16}{9 z}\right\}
\end{aligned}
$$

with $C_{A}=N_{c}=3, C_{F}=\left(N_{c}^{2}-1\right) /\left(2 N_{c}\right)=4 / 3$. The corresponding expressions in the DIS scheme are given in [21]. The class of basic functions is the same for both schemes.

Also in this case some of the functions $f_{i}(x)$ can be combined to somewhat simpler Mellin transforms. This is e.g. the case for the combination

$$
8 \mathrm{Li}_{2}(-z) \ln (1+z)+4 \ln z \ln ^{2}(1+z)+8 \mathrm{~S}_{1,2}(-z)
$$

in $c_{L, q}^{(2), N S}(z)$. These terms can be transformed using the relation

$$
\begin{aligned}
\int_{0}^{1} d z & z^{N-1}\left[2 \operatorname{Li}_{2}(-z) \ln (1+z)+\ln z \ln ^{2}(1+z)\right. \\
& \left.+2 \mathrm{~S}_{1,2}(-z)\right] \\
= & \frac{1}{N} \int_{0}^{1} d z z^{N}\left[\frac{\widetilde{\Phi}(z)}{1+z}-\frac{1}{2} \frac{\ln ^{2} z}{1+z}+\zeta(2) \frac{1}{1+z}\right] \\
& -\frac{\zeta(2)}{N} \ln 2+\frac{\zeta(3)}{4 N} .
\end{aligned}
$$


TABLE I. Numerical values of the coefficients $B_{2, j}(N)$ in Eq. (35).

\begin{tabular}{rccc}
\hline \hline$N$ & $B_{2, F}(N)$ & $B_{2, A}(N)$ & $B_{2, N_{F}}(N)$ \\
\hline 2 & 17.9078876005 & -3.53561968276 & -3.99999999998 \\
4 & 21.4337645358 & 31.8422327506 & -12.7409351851 \\
6 & 49.6093136699 & 58.4783326748 & -21.0097878018 \\
8 & 76.6379137872 & 92.3938671695 & -28.4436170993 \\
10 & 101.906494546 & 130.266385188 & -35.1459888118 \\
12 & 125.462647526 & 170.288348629 & -41.2458493431 \\
14 & 147.482822156 & 211.433606893 & -46.8491181970 \\
16 & 168.152110570 & 253.091881557 & -52.0380522803 \\
18 & 187.635850771 & 294.886826041 & -56.8764477704 \\
20 & 206.075234490 & 336.580388240 & -61.4143760545 \\
\hline \hline
\end{tabular}

In the coefficient function $c_{2}^{(2) \mathrm{NS}}(x)$ [22] and similar quantities [23-25] even more complicated functions $f_{i}(x)$ such as

$$
\frac{\log (x)}{1+x} \log (1+x) \log (1-x)
$$

or

$$
\frac{1}{1+x}\left[\operatorname{Li}_{3}\left(\frac{1-x}{1+x}\right)-\mathrm{Li}_{3}\left(-\frac{1-x}{1+x}\right)\right]
$$

emerge up to two-loop level. The full set of about 80 functions $f_{i}(x)$ and their Mellin transforms is given in the Appendix in tabular form in terms of the linear representations derived in Sec. IV.

All Mellin transforms can be expressed in terms of linear combinations of up to threefold sums. The fourfold sums, which emerge in intermediary steps, are found to be reducible to more elementary sums by algebaric relations, cf. Sec. V. From this representation the Mellin transforms of the different splitting functions, coefficient functions and hard scattering cross sections for space and time-like unpolarized and polarized processes in massless QED and QCD up to two-loop order can be assembled directly. The expressions apply for integer values of the Mellin index $N$. The analytic continuations to complex values of $N$ are derived in Ref. [43]. For a computer code containing these quantities see Ref. [2](c).

The explicit expressions for the Mellin transforms of the individual two-loop quantities are rather lengthly. Rather than giving the explicit expressions for the different coefficient functions we are going to provide the Mellin transforms of the contributing functions $f_{i}(x)$. This allows to calculate also the Mellin transform for an even wider class of processes.

An example which covers the more complicated Mellin transforms for the functions $f_{i}(x)$ is the coefficient function $c_{2}^{(2) \mathrm{NS}}(x)$, which was also studied in Ref. [44] recently.

We performed a numerical comparison of our representation with that given in [44] for the first 20 moments and find agreement (see Table I):

$$
c_{2}^{(2), \mathrm{NS}}(N)=C_{F}^{2} B_{2, F}(N)+C_{F} C_{A} B_{2, A}(N)+C_{F} N_{F} B_{2, N_{F}}(N) .
$$

The numbers were also compared with the first 10 moments given in Ref. [22] and the analytic results in Refs. [41].

\section{LINEAR REPRESENTATIONS OF HARMONIC SUMS BY MELLIN TRANSFORMS}

In the following we give explicit representations of all finite harmonic sums up to level four in terms of a linear combination between the Mellin transform of a Nielsen function of the same level and harmonic sums of lower level. Contrary to the algebraic relations being discussed in Sec. V we call these representations linear. They are obtained by consecutive partial integration starting from the integral representation Eqs. (14)-(17). The Mellin transforms of the individual functions $f_{i}(x)$ of the various two-loop quantities given in the Appendix will be expressed in terms of these harmonic sums. Up to two-loop order not all the sums are emerging. They will, however, contribute to expressions at higher loop level, similarly as in the case of lower level sums in the cases discussed below. We add as well the analytic continuations for the most simple cases, in which they can be expressed by the functions $\psi^{(k)}(z)$ and $\beta^{(k)}(z)$, where

$$
\begin{aligned}
& \psi(x)=\frac{1}{\Gamma(x)} \frac{d}{d x} \Gamma(x) \\
& \beta(x)=\frac{1}{2}\left[\psi\left(\frac{x+1}{2}\right)-\psi\left(\frac{x}{2}\right)\right] .
\end{aligned}
$$

As a shorthand notation we denote by $F_{1}(x)$ the following combination of Nielsen functions

$$
\begin{aligned}
F_{1}(x)= & \mathrm{S}_{1,2}\left(\frac{1-x}{2}\right)+\mathrm{S}_{1,2}(1-x)-\mathrm{S}_{1,2}\left(\frac{1-x}{1+x}\right) \\
& +\mathrm{S}_{1,2}\left(\frac{1}{1+x}\right)-\log (2) \operatorname{Li}_{2}\left(\frac{1-x}{2}\right) \\
& +\frac{1}{2} \log ^{2}(2) \log \left(\frac{1+x}{2}\right)-\log (2) \operatorname{Li}_{2}\left(\frac{1-x}{1+x}\right),
\end{aligned}
$$

which contributes to a series of harmonic sums given below.

The linear representation of the harmonic sums up to level four are given in the following. Here we define $\mathbf{M}[f](N)$ $=\int_{0}^{1} d x x^{N} f(x)$.

\section{A. First order sums}

The first order sums are

$$
\begin{aligned}
S_{-1}(N) & =(-1)^{N} \mathbf{M}\left[\frac{1}{1+x}\right](N)-\log (2) \\
& =(-1)^{N} \beta(N+1)-\log (2)
\end{aligned}
$$




$$
\begin{aligned}
S_{1}(N) & =\mathbf{M}\left[\left(\frac{1}{x-1}\right)_{+}\right](N) \\
& =\psi(N+1)+\gamma_{E} .
\end{aligned}
$$

\section{B. Second order sums}

The second order sums are

$$
\begin{aligned}
& S_{-2}(N)=(-1)^{N+1} \mathbf{M}\left[\frac{\log x}{1+x}\right](N)-\frac{1}{2} \zeta(2) \\
& =(-1)^{N+1} \beta^{\prime}(N+1)-\frac{1}{2} \zeta(2) \\
& S_{2}(N)=-\mathbf{M}\left[\frac{\log x}{x-1}\right](N)+\zeta(2) \\
& =-\psi^{\prime}(N+1)+\zeta(2) \\
& S_{-1,-1}(N)=-\mathbf{M}\left[\left(\frac{\log (1+x)}{x-1}\right)_{+}\right](N)+\log (2) \\
& \times\left[S_{1}(N)-S_{-1}(N)\right] \\
& =\frac{1}{2}\left\{\left[\beta(N+1)-(-1)^{N} \log (2)\right]^{2}\right. \\
& \left.+\zeta(2)-\psi^{\prime}(N+1)\right\} \\
& S_{-1,1}(N)=(-1)^{N+1} \mathbf{M}\left[\frac{\log (1-x)}{1+x}\right](N) \\
& -\frac{1}{2}\left[\zeta(2)-\log ^{2}(2)\right] \\
& =(-1)^{N} \mathbf{M}\left[\frac{\log (1+x)}{1+x}\right](N) \\
& +S_{1}(N) S_{-1}(N)+S_{-2}(N) \\
& +\left[S_{1}(N)-S_{-1}(N)\right] \log (2)-\frac{1}{2} \log ^{2}(2)
\end{aligned}
$$

$$
\begin{aligned}
S_{1,-1}(N)= & (-1)^{N+1} \mathbf{M}\left[\frac{\log (1+x)}{1+x}\right](N) \\
& -\left[S_{1}(N)-S_{-1}(N)\right] \log (2)+\frac{1}{2} \log ^{2}(2)
\end{aligned}
$$

$$
\begin{aligned}
S_{1,1}(N) & =-\mathbf{M}\left[\left(\frac{\log (1-x)}{x-1}\right){ }_{+}\right](N) \\
& =\frac{1}{2}\left\{\left[\psi(N+1)+\gamma_{E}\right]^{2}+\zeta(2)-\psi^{\prime}(N+1)\right\} .
\end{aligned}
$$

Here $\gamma_{E}$ denotes the Mascheroni number.

\section{Third order sums}

The third order sums are

$$
\begin{aligned}
S_{-3}(N)= & (-1)^{N} \frac{1}{2} \mathbf{M}\left[\frac{\log ^{2} x}{1+x}\right](N)-\frac{3}{4} \zeta(3) \\
= & (-1)^{N} \frac{1}{2} \beta^{\prime \prime}(N+1)-\frac{3}{4} \zeta(3) \\
S_{3}(N)= & \frac{1}{2} \mathbf{M}\left[\frac{\log ^{2} x}{x-1}\right](N)+\zeta(3) \\
= & \frac{1}{2} \psi^{\prime \prime}(N+1)+\zeta(3) \\
S_{-2,-1}(N)= & -\mathbf{M}\left[\left(\frac{\operatorname{Li}_{2}(-x)}{x-1}\right)_{+}\right](N) \\
& +\log (2)\left[S_{2}(N)-S_{-2}(N)\right] \\
& -\frac{\zeta(2)}{2} S_{1}(N)
\end{aligned}
$$

$$
\begin{aligned}
S_{-2,1}(N)= & (-1)^{N+1} \mathbf{M}\left[\frac{\operatorname{Li}_{2}(x)}{1+x}\right](N)+\zeta(2) S_{-1}(N) \\
& -\frac{5}{8} \zeta(3)+\zeta(2) \log (2)
\end{aligned}
$$

$$
\begin{aligned}
S_{2,-1}(N)= & (-1)^{N+1} \mathbf{M}\left[\frac{\operatorname{Li}_{2}(-x)}{1+x}\right](N)-\log (2) \\
& \times\left[S_{2}(N)-S_{-2}(N)\right]-\frac{1}{2} \zeta(2) S_{-1}(N) \\
& +\frac{1}{4} \zeta(3)-\frac{1}{2} \zeta(2) \log (2) \\
S_{2,1}(N)= & \mathbf{M}\left[\left(\frac{\operatorname{Li}_{2}(x)}{x-1}\right)_{+}\right](N)+\zeta(2) S_{1}(N)
\end{aligned}
$$

$$
\begin{aligned}
S_{-1,-2}(N)= & \mathbf{M}\left\{\left[\frac{\log (1+x) \log (x)+\mathrm{Li}_{2}(-x)}{x-1}\right]_{+}\right\}(N) \\
& +\frac{1}{2} \zeta(2)\left[S_{1}(N)-S_{-1}(N)\right]
\end{aligned}
$$




$$
\begin{aligned}
& S_{-1,2}(N)=(-1)^{N+1} \mathbf{M}\left[\frac{\operatorname{Li}_{2}(1-x)}{1+x}\right](N)+\zeta(2) S_{-1}(N)-\zeta(3)+\frac{3}{2} \zeta(2) \log (2) \\
& S_{1,-2}(N)=(-1)^{N} \mathbf{M}\left[\frac{\log (x) \log (1+x)+\operatorname{Li}_{2}(-x)}{1+x}\right](N)-\frac{1}{2} \zeta(2)\left[S_{1}(N)-S_{-1}(N)\right]-\frac{1}{8} \zeta(3)+\frac{1}{2} \zeta(2) \log (2) \\
& S_{1,2}(N)=-\mathbf{M}\left[\frac{\operatorname{Li}_{2}(1-x)}{x-1}\right](N)+\zeta(2) S_{1}(N)-\zeta(3) \\
& S_{-1,-1,-1}(N)=(-1)^{N+1} \mathbf{M}\left[\frac{\operatorname{Li}_{2}[(1-x) / 2]-\log (2) \log (1-x)}{1+x}\right](N)+\log (2)\left[S_{-1,1}(N)-S_{-1,-1}(N)\right] \\
& +\frac{1}{2}\left[\zeta(2)-\log ^{2}(2)\right] S_{-1}(N)-\frac{1}{4} \zeta(3)+\zeta(2) \log (2)-\frac{2}{3} \log ^{3}(2) \\
& =\frac{1}{6}\left[(-1)^{N} \beta(N+1)-\log (2)\right]^{3}-\frac{1}{2}\left[(-1)^{N} \beta(N+1)-\log (2)\right]\left[\psi^{\prime}(N+1)-\zeta(2)\right] \\
& +\frac{1}{3}\left[(-1)^{N} \frac{1}{2} \beta^{\prime \prime}(N+1)-\frac{3}{4} \zeta(3)\right] \\
& S_{-1,-1,1}(N)=\mathbf{M}\left\{\left[\frac{1}{x-1}\left(\log (1-x) \log \left(\frac{1+x}{2}\right)+\operatorname{Li}_{2}\left(\frac{1-x}{2}\right)\right)\right]_{+}\right\}(N)-\frac{1}{2}\left[\zeta(2)-\log ^{2}(2)\right] S_{-1}(N) \\
& S_{-1,1,-1}(N)=\frac{1}{2} \mathbf{M}\left\{\left[\frac{\log ^{2}(1+x)}{x-1}\right]_{+}\right\}(N)+\log (2)\left[S_{-1,-1}(N)-S_{-1,1}(N)\right]-\frac{1}{2} \log ^{2}(2)\left[S_{1}(N)-S_{-1}(N)\right] \\
& S_{-1,1,1}(N)=\frac{1}{2}(-1)^{N} \mathbf{M}\left[\frac{\log ^{2}(1-x)}{1+x}\right](N)-\frac{7}{8} \zeta(3)+\frac{1}{2} \zeta(2) \log (2)-\frac{1}{6} \log ^{3}(2) \\
& S_{1,-1,-1}(N)=\mathbf{M}\left\{\left[\frac{1}{x-1}\left(\log \left(\frac{1-x}{2}\right) \log (1+x)+\operatorname{Li}_{2}\left(\frac{1+x}{2}\right)\right)\right]_{+}\right\}(N)+\log (2)\left[S_{1,1}(N)-S_{1,-1}(N)\right] \\
& -\frac{1}{2}\left[\zeta(2)-\log ^{2}(2)\right] S_{1}(N) \\
& S_{1,-1,1}(N)=(-1)^{N} \mathbf{M}\left\{\frac{1}{1+x}\left[\log \left(\frac{1+x}{2}\right) \log (1-x)+\operatorname{Li}_{2}\left(\frac{1-x}{2}\right)\right]\right\}(N)-\frac{1}{2}\left[\zeta(2)-\log ^{2}(2)\right] S_{1}(N) \\
& +\frac{1}{8} \zeta(3)-\frac{1}{2} \zeta(2) \log (2)+\frac{1}{3} \log ^{3}(2) \\
& S_{1,1,-1}(N)=(-1)^{N} \frac{1}{2} \mathbf{M}\left[\frac{\log ^{2}(1+x)}{1+x}\right](N)+\log (2)\left[S_{1,-1}(N)-S_{1,1}(N)\right]+\frac{1}{2} \log ^{2}(2)\left[S_{1}(N)-S_{-1}(N)\right]-\frac{1}{6} \log ^{3}(2) \\
& S_{1,1,1}(N)=\frac{1}{2} \mathbf{M}\left[\left(\frac{\log ^{2}(1-x)}{x-1}\right)_{+}\right](N) \\
& =\frac{1}{6}\left[\psi(N+1)+\gamma_{E}\right]^{3}-\frac{1}{2}\left[\psi(N+1)+\gamma_{E}\right]\left[\psi^{\prime}(N+1)-\zeta(2)\right]+\frac{1}{3}\left[\frac{1}{2} \psi^{\prime \prime}(N+1)+\zeta(3)\right] .
\end{aligned}
$$




\section{Fourth order sums}

The fourth order sums are

$$
\begin{aligned}
& S_{-4}(N)=(-1)^{N+1} \frac{1}{6} \mathbf{M}\left[\frac{\log ^{3} x}{1+x}\right](N)-\frac{7}{20} \zeta^{2}(2) \\
& =(-1)^{N+1} \frac{1}{6} \beta^{(3)}(N+1)-\frac{7}{20} \zeta^{2}(2) \\
& S_{4}(N)=-\frac{1}{6} \mathbf{M}\left[\frac{\log ^{3} x}{x-1}\right](N)+\frac{2}{5} \zeta^{2}(2) \\
& =-\frac{1}{6} \psi^{(3)}(N+1)+\frac{2}{5} \zeta^{2}(2) \\
& S_{-2,-2}(N)=\mathbf{M}\left\{\left[\frac{1}{x-1}\left(\log (x) \operatorname{Li}_{2}(-x)-2 \operatorname{Li}_{3}(-x)\right)\right]_{+}\right\}(N)+\frac{1}{2} \zeta(2)\left[S_{2}(N)-S_{-2}(N)\right]-\frac{3}{2} S_{1}(N) \\
& =\frac{1}{2}\left\{\left[\beta^{\prime}(N+1)+(-1)^{N} \frac{1}{2} \zeta(2)\right]^{2}-\frac{1}{6} \psi^{(3)}(N+1)+\frac{2}{5} \zeta(2)^{2}\right\} \\
& S_{-2,2}(N)=(-1)^{N+1} \mathbf{M}\left\{\frac{1}{1+x}\left[2 \operatorname{Li}_{3}(x)-\log (x)\left(\operatorname{Li}_{2}(x)+\zeta(2)\right)\right]\right\}(N)+\zeta(2) S_{-2}(N)+2 \zeta(3) S_{-1}(N)+\frac{71}{40} \zeta(2)^{2} \\
& -4 \mathrm{Li}_{4}\left(\frac{1}{2}\right)-\frac{3}{2} \zeta(3) \log (2)+\zeta(2) \log ^{2}(2)-\frac{1}{6} \log ^{4}(2) \\
& S_{2,-2}(N)=(-1)^{N} \mathbf{M}\left\{\frac{1}{1+x}\left[\operatorname{Li}_{2}(-x) \log (x)-2 \operatorname{Li}_{3}(-x)\right]\right\}(N)-\frac{3}{2} \zeta(3) S_{-1}(N)+\frac{1}{2} \zeta(2)\left[S_{-2}(N)-S_{2}(N)\right]-\frac{11}{8} \zeta(2)^{2} \\
& +4 \operatorname{Li}_{4}\left(\frac{1}{2}\right)+2 \zeta(3) \log (2)-\zeta(2) \log ^{2}(2)+\frac{1}{6} \log ^{4}(2) \\
& S_{2,2}(N)=\mathbf{M}\left[\left(\frac{\log (x)\left[\mathrm{Li}_{2}(x)+\zeta(2)\right]-2 \mathrm{Li}_{3}(x)}{x-1}\right)_{+}\right](N)+\zeta(2) S_{2}(N)+2 \zeta(3) S_{1}(N) \\
& =\frac{1}{2}\left\{\left[\psi^{\prime}(N+1)-\zeta(2)\right]^{2}-\frac{1}{6} \psi^{(3)}(N+1)+\frac{2}{5} \zeta(2)^{2}\right\} \\
& S_{-3,-1}(N)=\mathbf{M}\left[\left(\frac{\mathrm{Li}_{3}(-x)}{x-1}\right)_{+}\right](N)+\log (2)\left[S_{3}(N)-S_{-3}(N)\right]-\frac{1}{2} \zeta(2) S_{2}(N)+\frac{3}{4} \zeta(3) S_{1}(N) \\
& S_{-3,1}(N)=(-1)^{N} \mathbf{M}\left[\frac{\mathrm{Li}_{3}(x)}{1+x}\right](N)+\zeta(2) S_{-2}(N)-\zeta(3) S_{-1}(N)-\frac{3}{5} \zeta(2)^{2}+2 \operatorname{Li}_{4}\left(\frac{1}{2}\right)+\frac{3}{4} \zeta(3) \log (2)-\frac{1}{2} \zeta(2) \log { }^{2}(2) \\
& +\frac{1}{12} \log ^{4}(2) \\
& S_{3,-1}(N)=(-1)^{N} \mathbf{M}\left[\frac{\operatorname{Li}_{3}(-x)}{1+x}\right](N)+\log (2)\left[S_{-3}(N)-S_{3}(N)\right] \\
& -\frac{1}{2} \zeta(2) S_{-2}(N)+\frac{3}{4} \zeta(3) S_{-1}(N)-\frac{1}{8} \zeta(2)^{2}+\frac{3}{4} \zeta(3) \log (2) \\
& S_{3,1}(N)=\frac{1}{2} \mathbf{M}\left[\frac{\operatorname{Li}_{2}(x)}{x-1} \log (x)\right](N)+\zeta(2) S_{2}(N)-\frac{1}{4} S_{2}^{2}(N)-\frac{1}{4} S_{4}(N)-\frac{3}{20} \zeta^{2}(2)
\end{aligned}
$$




$$
\begin{aligned}
& S_{-1,-3}(N)=-\frac{1}{2} \mathbf{M}\left\{\left[\frac{1}{x-1}\left(\log ^{2}(x) \log (1+x)+2 \log (x) \operatorname{Li}_{2}(-x)-2 \operatorname{Li}_{3}(-x)\right)\right]_{+}\right\}(N)+\frac{3}{4} \zeta(3)\left[S_{1}(N)-S_{-1}(N)\right] \\
& S_{-1,3}(N)=(-1)^{N+1} \mathbf{M}\left[\frac{S_{1,2}(1-x)}{1+x}\right](N)+\zeta(3) S_{-1}(N)-\frac{19}{40} \zeta^{2}(2)+\frac{7}{4} \zeta(3) \log (2) \\
& S_{1,-3}(N)=\frac{(-1)^{N}}{2} \mathbf{M}\left\{\frac{1}{1+x}\left[2 \operatorname{Li}_{3}(-x)-\log ^{2}(x) \log (1+x)-2 \log (x) \operatorname{Li}_{2}(-x)\right]\right\}(N)+\frac{3}{4} \zeta(3)\left[S_{-1}(N)-S_{1}(N)\right] \\
& +\frac{3}{4} \zeta(2)^{2}-2 \operatorname{Li}_{4}\left(\frac{1}{2}\right)-\zeta(3) \log (2)+\frac{1}{2} \zeta(2) \log ^{2}(2)-\frac{1}{12} \log ^{4}(2) \\
& S_{1,3}(N)=-\frac{1}{2} \mathbf{M}\left\{\left[\frac{1}{x-1}\left(\log ^{2}(x) \log (1-x)+2 \log (x) \operatorname{Li}_{2}(x)-2 \operatorname{Li}_{3}(x)\right)\right]_{+}\right\}(N) \\
& S_{-2,-1,-1}(N)=(-1)^{N} \mathbf{M}\left\{\frac{1}{1+x}\left[F_{1}(x)-\log (x) \operatorname{Li}_{2}\left(\frac{1+x}{2}\right)\right]\right\}(N)+(-1)^{N+1} \mathbf{M}\left\{\frac{1}{1+x} \log (x) \log (1+x) \log \left(\frac{1-x}{2}\right)\right\}(N) \\
& +\log (2)\left[S_{-2,1}(N)-S_{-2,-1}(N)\right]-\frac{1}{2}\left[\zeta(2)-\log ^{2}(2)\right] S_{-2}(N)-\left[\frac{1}{8} \zeta(3)-\frac{1}{6} \log ^{3}(2)\right] S_{-1}(N)-4 \operatorname{Li}_{4}\left(\frac{1}{2}\right) \\
& +\frac{23}{20} \zeta(2)^{2}-\frac{11}{4} \zeta(3) \log (2)+\frac{5}{4} \zeta(2) \log ^{2}(2) \\
& S_{-2,-1,1}(N)=-\mathbf{M}\left\{\left[\frac{1}{x-1}\left(F_{1}(x)+\frac{1}{2} \mathrm{~S}_{1,2}\left(x^{2}\right)-\mathrm{S}_{1,2}(-x)-\mathrm{S}_{1,2}(x)\right)\right]_{+}\right\}(N)-\mathbf{M}\left\{\left[\frac { 1 } { x - 1 } \operatorname { l o g } ( x ) \left(\operatorname{Li}_{2}\left(\frac{1-x}{2}\right)\right.\right.\right. \\
& \left.-\log (2) \log (1-x))]_{+}\right\}(N)-\frac{1}{2}\left[\zeta(2)-\log ^{2}(2)\right] S_{-2}(N)-\left[\frac{1}{2} \zeta(3)+\frac{1}{6} \log ^{3}(2)\right] S_{1}(N) \\
& S_{-2,1,-1}(N)=-\mathbf{M}\left\{\left[\frac{1}{x-1} \mathrm{~S}_{1,2}(-x)\right]_{+}\right\}(N)-\left[S_{-2,1}(N)-S_{-2,-1}(N)\right] \log (2)+\left[S_{-2}(N)-S_{2}(N)\right] \frac{1}{2} \log ^{2}(2)+\frac{1}{8} \zeta(3) S_{1}(N) \\
& S_{-2,1,1}(N)=(-1)^{N+1} \mathbf{M}\left[\frac{S_{1,2}(x)}{1+x}\right](N)+\zeta(3) S_{-1}(N)-\operatorname{Li}_{4}\left(\frac{1}{2}\right)+\frac{1}{8} \zeta^{2}(2)+\frac{1}{8} \zeta(3) \log (2)+\frac{1}{4} \zeta(2) \log ^{2}(2)-\frac{1}{24} \log ^{4}(2) \\
& S_{2,-1,-1}(N)=\mathbf{M}\left\{\left[\frac{1}{x-1}\left(F_{1}(x)-\log (x) \operatorname{Li}_{2}\left(\frac{1+x}{2}\right)\right)\right]_{+}\right\}(N)-\mathbf{M}\left\{\left[\frac{1}{x-1} \log (x) \log (1+x) \log \left(\frac{1-x}{2}\right)\right]_{+}\right\}(N) \\
& +\log (2)\left[S_{2,1}(N)-S_{2,-1}(N)\right]-\frac{1}{2}\left[\zeta(2)-\log ^{2}(2)\right] S_{2}(N)-\left[\frac{1}{8} \zeta(3)-\frac{1}{6} \log ^{3}(2)\right] S_{1}(N) \\
& S_{2,-1,1}(N)=(-1)^{N+1} \mathbf{M}\left\{\frac{1}{x+1}\left[F_{1}(x)+\log (x)\left(\operatorname{Li}_{2}\left(\frac{1-x}{2}\right)-\log (2) \log (1-x)\right)\right]\right\}(N) \\
& +(-1)^{N+1} \mathbf{M}\left\{\frac{1}{x+1}\left[\frac{1}{2} \mathrm{~S}_{1,2}\left(x^{2}\right)-\mathrm{S}_{1,2}(x)-\mathrm{S}_{1,2}(-x)\right]\right\}(N)-\frac{1}{2}\left[\zeta(2)-\log ^{2}(2)\right] S_{2}(N)-\left[\frac{1}{2} \zeta(3)\right. \\
& \left.+\frac{1}{6} \log ^{3}(2)\right] S_{-1}(N)+4 \operatorname{Li}_{4}\left(\frac{1}{2}\right)-\frac{3}{2} \zeta(2)^{2}+\frac{17}{8} \zeta(3) \log (2)-\frac{3}{4} \zeta(2) \log ^{2}(2)
\end{aligned}
$$




$$
\begin{aligned}
& S_{2,1,-1}(N)=(-1)^{N+1} \mathbf{M}\left[\frac{S_{1,2}(-x)}{1+x}\right](N)-\log (2)\left[S_{2,1}(N)-S_{2,-1}(N)\right]+\frac{1}{2} \log ^{2}(2)\left[S_{2}(N)-S_{-2}(N)\right]+\frac{1}{8} \zeta(3) S_{-1}(N) \\
& +3 \operatorname{Li}_{4}\left(\frac{1}{2}\right)-\frac{6}{5} \zeta^{2}(2)+\frac{11}{4} \zeta(3) \log (2)-\frac{3}{4} \zeta(2) \log ^{2}(2)+\frac{1}{8} \log ^{4}(2) \\
& S_{2,1,1}(N)=-\mathbf{M}\left[\left(\frac{\mathrm{S}_{1,2}(x)}{x-1}\right)_{+}\right](N)+\zeta(3) S_{1}(N) \\
& S_{-1,-2,-1}(N)=(-1)^{N} \mathbf{M}\left\{\frac{1}{1+x}\left[\operatorname{Li}_{2}(-x) \log (1-x)+\frac{1}{2} \mathrm{~S}_{1,2}\left(x^{2}\right)-\mathrm{S}_{1,2}(x)-\mathrm{S}_{1,2}(-x)\right]\right\}(N)+\log (2)\left[S_{-1,2}(N)-S_{-1,-2}(N)\right] \\
& -\frac{1}{2} \zeta(2) S_{-1,1}(N)-\frac{33}{20} \zeta(2)^{2}+4 \operatorname{Li}_{4}\left(\frac{1}{2}\right)+\frac{13}{4} \zeta(3) \log (2)-\frac{3}{4} \zeta(2) \log ^{2}(2)+\frac{1}{6} \log ^{4}(2) \\
& S_{-1,-2,1}(N)=\mathbf{M}\left\{\left[\frac{\log (1+x) \operatorname{Li}_{2}(x)+\mathrm{S}_{1,2}\left(x^{2}\right) / 2-\mathrm{S}_{1,2}(x)-\mathrm{S}_{1,2}(-x)}{x-1}\right]_{+}\right\}(N)+\zeta(2) S_{-1,-1}(N)+\left[\frac{5}{8} \zeta(3)-\zeta(2) \log (2)\right] \\
& \times\left[S_{1}(N)-S_{-1}(N)\right] \\
& S_{-1,2,-1}(N)=\mathbf{M}\left[\left(\frac{\log (1+x) \mathrm{Li}_{2}(-x)+2 \mathrm{~S}_{1,2}(-x)}{x-1}\right)_{+}\right](N)+\log (2)\left[S_{-1,-2}(N)-S_{-1,2}(N)\right]-\frac{1}{2} \zeta(2) S_{-1,-1}(N) \\
& +\left[\frac{1}{4} \zeta(3)-\frac{1}{2} \zeta(2) \log (2)\right]\left[S_{-1}(N)-S_{1}(N)\right] \\
& S_{-1,2,1}(N)=(-1)^{N} \mathbf{M}\left\{\frac{1}{1+x}\left[\operatorname{Li}_{2}(x) \log (1-x)+2 \mathrm{~S}_{1,2}(x)\right]\right\}(N)+\zeta(2) S_{-1,1}(N)+\frac{19}{40} \zeta(2)^{2}-\operatorname{Li}_{4}\left(\frac{1}{2}\right)-\frac{1}{4} \zeta(3) \log (2) \\
& -\frac{1}{4} \zeta(2) \log ^{2}(2)-\frac{1}{24} \log ^{4}(2) \\
& S_{1,-2,-1}(N)=\mathbf{M}\left\{\left[\frac{1}{x-1} \log (1-x) \mathrm{Li}_{2}(-x)\right]_{+}\right\}(N)+\mathbf{M}\left\{\left[\frac{1}{x-1}\left(\frac{1}{2} \mathrm{~S}_{1,2}\left(x^{2}\right)-\mathrm{S}_{1,2}(x)-\mathrm{S}_{1,2}(-x)\right)\right]_{+}\right\}(N) \\
& +\log (2)\left[\mathrm{S}_{1,2}(N)-S_{1,-2}(N)\right]-\frac{1}{2} \zeta(2) S_{1,1}(N) \\
& S_{1,-2,1}(N)=(-1)^{N} \mathbf{M}\left\{\frac{1}{1+x}\left[\operatorname{Li}_{2}(x) \log (1+x)+\frac{1}{2} \mathrm{~S}_{1,2}\left(x^{2}\right)-\mathrm{S}_{1,2}(x)-\mathrm{S}_{1,2}(-x)\right]\right\}(N)+\zeta(2) S_{1,-1}(N)+[\zeta(2) \log (2) \\
& \left.-\frac{5}{8} \zeta(3)\right]\left[S_{1}(N)-S_{-1}(N)\right]-\frac{3}{40} \zeta(2)^{2}+\frac{5}{8} \zeta(3) \log (2)-\frac{1}{2} \zeta(2) \log ^{2}(2) \\
& S_{1,2,-1}(N)=(-1)^{N} \mathbf{M}\left\{\frac{1}{1+x}\left[\operatorname{Li}_{2}(-x) \log (1+x)+2 \mathrm{~S}_{1,2}(-x)\right]\right\}(N)-\log (2)\left[\mathrm{S}_{1,2}(N)-S_{1,-2}(N)\right]-\frac{1}{2} \zeta(2) S_{1,-1}(N) \\
& +\left[\frac{1}{4} \zeta(3)-\frac{1}{2} \zeta(2) \log (2)\right]\left[S_{1}(N)-S_{-1}(N)\right]+\frac{6}{5} \zeta(2)^{2}-3 \operatorname{Li}_{4}\left(\frac{1}{2}\right)-\frac{23}{8} \zeta(3) \log (2)+\zeta(2) \log ^{2}(2)-\frac{1}{8} \log ^{4}(2) \\
& S_{1,2,1}(N)=\mathbf{M}\left\{\left[\frac{1}{x-1}\left(\operatorname{Li}_{2}(x) \log (1-x)+2 \mathrm{~S}_{1,2}(x)\right)\right]_{+}\right\}(N)+\zeta(2) S_{1,1}(N)
\end{aligned}
$$




$$
\begin{aligned}
& S_{-1,-1,-2}(N)=(-1)^{N+1} \mathbf{M}\left\{\frac{1}{1+x}\left[F_{1}(x)+\log (1-x) \operatorname{Li}_{2}(-x)\right]\right\}(N) \\
& +(-1)^{N+1} \mathbf{M}\left\{\frac{1}{1+x}\left[\frac{1}{2} \mathrm{~S}_{1,2}\left(x^{2}\right)-\mathrm{S}_{1,2}(x)-\mathrm{S}_{1,2}(-x)\right]\right\}(N)+\frac{1}{2} \zeta(2)\left[S_{-1,1}(N)-S_{-1,-1}(N)\right] \\
& +\left[\frac{9}{8} \zeta(3)-\frac{3}{2} \zeta(2) \log (2)-\frac{1}{6} \log ^{3}(2)\right] S_{-1}(N)-\frac{1}{10} \zeta(2)^{2}+\frac{17}{8} \zeta(3) \log (2)-\frac{7}{4} \zeta(2) \log ^{2}(2)-\frac{1}{6} \log ^{4}(2) \\
& S_{-1,-1,2}(N)=\mathbf{M}\left\{\left[\frac{1}{x-1}\left(F_{1}(x)+\log (1+x) \operatorname{Li}_{2}(1-x)\right)\right]_{+}\right\}(N)+\zeta(2) S_{-1,-1}(N) \\
& -\left[\zeta(3)-\frac{3}{2} \zeta(2) \log (2)\right] S_{-1}(N)-\left[\frac{1}{8} \zeta(3)-\frac{1}{6} \log ^{3}(2)\right] S_{1}(N) \\
& S_{-1,1,-2}(N)=-\mathbf{M}\left\{\left[\frac{1}{x-1}\left(\operatorname{Li}_{2}(-x) \log (1+x)+\mathrm{S}_{1,2}(-x)+\frac{1}{2} \log (x) \log ^{2}(1+x)\right)\right]_{+}\right\}(N)-\frac{1}{2} \zeta(2)\left[S_{-1,1}(N)-S_{-1,-1}(N)\right] \\
& +\left[\frac{1}{8} \zeta(3)-\frac{1}{2} \zeta(2) \log (2)\right]\left[S_{1}(N)-S_{-1}(N)\right] \\
& S_{-1,1,2}(N)=(-1)^{N} \mathbf{M}\left[\frac{\operatorname{Li}_{3}(1-x)}{1+x}\right](N)+\zeta(2) S_{-1,1}(N)-\zeta(3) S_{-1}(N) \\
& -\mathrm{Li}_{4}\left(\frac{1}{2}\right)+\frac{9}{20} \zeta^{2}(2)-\frac{7}{8} \zeta(3) \log (2)-\frac{1}{2} \zeta(2) \log ^{2}(2)-\frac{1}{24} \log ^{4}(2) \\
& S_{1,-1,-2}(N)=-\mathbf{M}\left\{\left[\frac{1}{x-1}\left(F_{1}(x)+\log (1-x) \operatorname{Li}_{2}(-x)\right)\right]_{+}\right\}(N)-\mathbf{M}\left\{\left[\frac{1}{x-1}\left(\frac{1}{2} \mathrm{~S}_{1,2}\left(x^{2}\right)-\mathrm{S}_{1,2}(x)-\mathrm{S}_{1,2}(-x)\right)\right]_{+}\right\}(N) \\
& +\frac{1}{2} \zeta(2)\left[S_{1,1}(N)-S_{1,-1}(N)\right]+\left[\frac{9}{8} \zeta(3)-\frac{3}{2} \zeta(2) \log (2)-\frac{1}{6} \log ^{3}(2)\right] S_{1}(N) \\
& S_{1,-1,2}(N)=(-1)^{N} \mathbf{M}\left\{\frac{1}{1+x}\left[F_{1}(x)+\log (1+x) \operatorname{Li}_{2}(1-x)\right]\right\}(N)+\zeta(2) S_{1,-1}(N)-\left[\zeta(3)-\frac{3}{2} \zeta(2) \log (2)\right] S_{1}(N) \\
& -\left[\frac{1}{8} \zeta(3)-\frac{1}{6} \log ^{3}(2)\right] S_{-1}(N)+\frac{7}{8} \zeta(2)^{2}-2 \operatorname{Li}_{4}\left(\frac{1}{2}\right)-\frac{11}{4} \zeta(3) \log (2)+\frac{5}{4} \zeta(2) \log ^{2}(2)+\frac{1}{12} \log ^{4}(2) \\
& S_{1,1,-2}(N)=(-1)^{N+1} \mathbf{M}\left[\frac{\mathrm{S}_{1,2}(-x)+\log ^{2}(2) \log (x) / 2+\log (2) \operatorname{Li}_{2}(-x)}{1+x}\right](N) \\
& +\frac{1}{2} \zeta(2)\left[S_{1,-1}(N)-S_{1,1}(N)\right]-\left[\frac{1}{8} \zeta(3)-\frac{1}{2} \zeta(2) \log (2)\right]\left[S_{1}(N)-S_{-1}(N)\right]+\operatorname{Li}_{4}\left(\frac{1}{2}\right)-\frac{2}{5} \zeta(2)^{2}+\zeta(3) \log (2) \\
& -\frac{1}{2} \zeta(2) \log ^{2}(2)+\frac{1}{24} \log ^{4}(2) \\
& S_{1,1,2}(N)=\mathbf{M}\left[\frac{\mathrm{Li}_{3}(1-x)}{x-1}\right](N)+\zeta(2) S_{1,1}(N)-\zeta(3) S_{1}(N)+\frac{2}{5} \zeta^{2}(2)
\end{aligned}
$$




$$
\begin{aligned}
& S_{-1,-1,-1,-1}(N)=\mathbf{M}\left\{\left[\frac{1}{x-1}\left(\operatorname{Li}_{2}\left(\frac{1-x}{2}\right) \log \left(\frac{1+x}{4}\right)+2 S_{1,2}\left(\frac{1-x}{2}\right)\right)\right]_{+}\right\}(N) \\
& -\log (2) \mathbf{M}\left\{\left[\frac{1}{x-1}\left(\log (1-x) \log \left(\frac{1+x}{2}\right)\right)\right]_{+}\right\}(N) \\
& +\log (2)\left[S_{-1,-1,1}(N)-S_{-1,-1,-1}(N)\right]+\frac{1}{2}\left[\zeta(2)-\log ^{2}(2)\right] S_{-1,-1}(N) \\
& -\left[\frac{1}{4} \zeta(3)-\zeta(2) \log (2)+\frac{2}{3} \log ^{3}(2)\right] S_{-1}(N) \\
& =\frac{1}{24}\left[\beta(N+1)-(-1)^{N} \log (2)\right]^{4}-\frac{1}{4}\left[\beta(N+1)-(-1)^{N} \log (2)\right]^{2}\left[\psi^{\prime}(N+1)-\zeta(2)\right] \\
& +\frac{1}{3}\left[\beta(N+1)-(-1)^{N} \log (2)\right]\left[\beta^{\prime \prime}(N+1)-(-1)^{N} \frac{3}{4} \zeta(3)\right] \\
& +\frac{1}{8}\left[\psi^{\prime}(N+1)-\zeta(2)\right]^{2}-\frac{1}{4}\left[\frac{1}{6} \psi^{(3)}(N+1)-\frac{2}{5} \zeta(2)^{2}\right] \\
& S_{-1,-1,-1,1}(N)=(-1)^{N+1} \mathbf{M}\left\{\frac{1}{1+x}\left[2 \operatorname{Li}_{3}\left(\frac{1-x}{2}\right)-\log (1-x) \operatorname{Li}_{2}\left(\frac{1-x}{2}\right)\right]\right\}(N)+\left[\frac{7}{4} \zeta(3)-\zeta(2) \log (2)+\frac{1}{3} \log 3(2)\right] S_{-1}(N) \\
& -\frac{1}{2}\left[\zeta(2)-\log ^{2}(2)\right] S_{-1,-1}(N)-\frac{9}{40} \zeta(2)^{2}-\frac{3}{4} \zeta(2) \log ^{2}(2)+2 \zeta(3) \log (2)+\frac{1}{8} \log ^{4}(2) \\
& S_{1,-1,1,-1}(N)=-\mathbf{M}\left\{\left[\frac{1}{x-1} \mathrm{~S}_{1,2}\left(\frac{1-x}{2}\right)\right]_{+}\right\}(N)+\mathbf{M}\left\{\left[\frac{1}{x-1}\left(\log (2) \operatorname{Li}_{2}\left(\frac{1-x}{2}\right)-\frac{1}{2} \log ^{2}(2) \log \left(\frac{1-x}{2}\right)\right)\right]_{+}\right\}(N) \\
& +\log (2)\left[S_{1,-1,-1}(N)-S_{1,-1,1}(N)\right]-\frac{1}{2} \log ^{2}(2)\left[S_{1,1}(N)-S_{1,-1}(N)\right]+\left[\frac{1}{8} \zeta(3)-\frac{1}{2} \zeta(2) \log (2)\right. \\
& \left.-\frac{1}{6} \log ^{3}(2)\right] S_{1}(N) \\
& S_{-1,-1,1,1}(N)=-\mathbf{M}\left\{\left[\frac{\mathrm{S}_{1,2}[(1+x) / 2]+(1 / 2) \log (1+x) \log ^{2}(2)}{x-1}\right]_{+}\right\}(N)+\mathbf{M}\left\{\left[\frac{1}{x-1} \log (2) \operatorname{Li}_{2}\left(\frac{1+x}{2}\right)\right]_{+}\right\}(N) \\
& -\left[\frac{7}{8} \zeta(3)-\frac{1}{2} \zeta(2) \log (2)+\frac{1}{6} \log ^{3}(2)\right] S_{-1}(N)+\left[\zeta(3)-\log (2) \zeta(2)+\frac{1}{2} \log ^{3}(2)\right] S_{1}(N) \\
& S_{-1,1,-1,-1}(N)=(-1)^{N} \mathbf{M}\left\{\left[\frac{1}{x-1}\left(\operatorname{Li}_{3}\left(\frac{1-x}{2}\right)-\log \left(\frac{1-x}{2}\right)\right)\right]_{+}\right\}(N)-(-1)^{N^{1}} \frac{1}{2} \log (2) \mathbf{M}\left\{\left[\frac{1}{x-1} \log ^{2}\left(\frac{1-x}{2}\right)\right]_{+}\right\}(N) \\
& +\log (2)\left[S_{-1,1,1}(N)-S_{-1,1,-1}(N)\right]-\frac{1}{2}\left[\zeta(2)-\log ^{2}(2)\right] S_{-1,1}(N) \\
& -\left[\frac{7}{8} \zeta(3)+\frac{1}{2} \zeta(2) \log (2)-\frac{1}{3} \log ^{3}(2)\right] S_{-1}(N)-\frac{3}{8} \zeta(2)^{2}-\frac{1}{4} \zeta(2) \log ^{2}(2)+\frac{1}{8} \log ^{4}(2) \\
& S_{-1,1,-1,1}(N)=\mathbf{M}\left\{\left[\frac{1}{x-1}\left(\operatorname{Li}_{3}\left(\frac{1+x}{2}\right)-\zeta(2) \log \left(\frac{1+x}{2}\right)\right)\right]_{+}\right\}(N)-\mathbf{M}\left\{\left[\frac{1}{x-1} \frac{1}{2} \log ^{2}\left(\frac{1+x}{2}\right) \log (2)\right]_{+}\right\}(N) \\
& -\zeta(3) S_{1}(N)+\left[\frac{1}{8} \zeta(3)-\frac{1}{2} \zeta(2) \log (2)+\frac{1}{3} \log ^{3}(2)\right] S_{-1}(N)-\frac{1}{2}\left[\zeta(2)-\log ^{2}(2)\right] S_{-1,1}(N)
\end{aligned}
$$




$$
\begin{aligned}
& S_{-1,1,1,-1}(N)=-\frac{1}{6} \mathbf{M}\left\{\left[\frac{\log ^{3}(1+x)}{x-1}\right]_{+}\right\}(N)+\log (2)\left[S_{-1,1,-1}(N)-S_{-1,1,1}(N)\right] \\
& +\frac{1}{2} \log ^{2}(2)\left[S_{-1,1}(N)-S_{-1,-1}(N)\right]+\frac{1}{6} \log ^{3}(2)\left[S_{1}(N)-S_{-1}(N)\right] \\
& S_{-1,1,1,1}(N)=(-1)^{N+1} \frac{1}{6} \mathbf{M}\left[\frac{\log ^{3}(1-x)}{1+x}\right](N)-\operatorname{Li}_{4}\left(\frac{1}{2}\right) \\
& S_{1,-1,-1,-1}(N)=(-1)^{N} \mathbf{M}\left\{\frac{1}{1+x}\left[\operatorname{Li}_{2}\left(\frac{1-x}{2}\right) \log \left(\frac{1+x}{4}\right)+2 S_{1,2}\left(\frac{1-x}{2}\right)\right]\right\}(N) \\
& -(-1)^{N} \mathbf{M}\left\{\frac{1}{1+x} \log (2) \log (1-x) \log \left(\frac{1+x}{2}\right)\right\}(N)+\log (2)\left[S_{1,-1,1}(N)-S_{1,-1,-1}(N)\right] \\
& +\frac{1}{2}\left[\zeta(2)-\log ^{2}(2)\right] S_{1,-1}(N)-\left[\frac{1}{4} \zeta(3)-\zeta(2) \log (2)+\frac{2}{3} \log ^{3}(2)\right] S_{1}(N)+\frac{6}{5} \zeta(2)^{2} \\
& -3 \mathrm{Li}_{4}\left(\frac{1}{2}\right)-3 \zeta(3) \log (2)+\frac{3}{2} \zeta(2) \log ^{2}(2)-\frac{1}{2} \log ^{4}(2) \\
& S_{1,-1,-1,1}(N)=-\mathbf{M}\left\{\left[\frac{1}{x-1}\left(2 \operatorname{Li}_{3}\left(\frac{1-x}{2}\right)-\log (1-x) \operatorname{Li}_{2}\left(\frac{1-x}{2}\right)\right)\right]_{+}\right\}(N) \\
& +\left[\frac{7}{4} \zeta(3)-\zeta(2) \log (2)+\frac{1}{3} \log ^{3}(2)\right] S_{1}(N)-\frac{1}{2}\left[\zeta(2)-\log ^{2}(2)\right] S_{1,-1}(N) \\
& S_{-1,-1,1,-1}(N)=(-1)^{N+1} \mathbf{M}\left\{\frac{1}{1+x} \mathrm{~S}_{1,2}\left(\frac{1-x}{2}\right)\right\}(N)+(-1)^{N} \mathbf{M}\left\{\frac{1}{1+x}\left[\log (2) \operatorname{Li}_{2}\left(\frac{1-x}{2}\right)-\frac{1}{2} \log 2(2) \log \left(\frac{1-x}{2}\right)\right]\right\}(N) \\
& +\log (2)\left[S_{-1,-1,-1}(N)-S_{-1,-1,1}(N)\right]-\frac{1}{2} \log ^{2}(2)\left[S_{-1,1}(N)-S_{-1,-1}(N)\right]+\left[\frac{1}{8} \zeta(3)-\frac{1}{2} \zeta(2) \log (2)\right. \\
& \left.-\frac{1}{6} \log ^{3}(2)\right] S_{-1}(N)-\frac{6}{5} \zeta(2)^{2}+3 \operatorname{Li}_{4}\left(\frac{1}{2}\right)+3 \zeta(3) \log (2)-\frac{3}{2} \zeta(2) \log ^{2}(2) \\
& S_{1,-1,1,1}(N)=(-1)^{N+1} \frac{1}{2} \mathbf{M}\left\{\frac{\log [(1+x) / 2] \log ^{2}(1-x)}{1+x}\right\}(N)+(-1)^{N+1} \mathbf{M}\left\{\frac{\log (1-x) \operatorname{Li}_{2}[(1-x) / 2]}{1+x}\right\}(N) \\
& +(-1)^{N} \mathbf{M}\left\{\frac{\operatorname{Li}_{3}[(1-x) / 2]}{1+x}\right\}(N)-\left[\frac{7}{8} \zeta(3)-\frac{1}{2} \zeta(2) \log (2)+\frac{1}{6} \log ^{3}(2)\right] S_{1}(N)-\zeta(3) \log (2)-\frac{1}{8} \log ^{4}(2) \\
& +\frac{1}{20} \zeta(2)^{2}+\frac{1}{2} \zeta(2) \log ^{2}(2) \\
& S_{1,1,-1,-1}(N)=\mathbf{M}\left\{\left[\frac{1}{x-1}\left(\operatorname{Li}_{3}\left(\frac{1-x}{2}\right)-\log \left(\frac{1-x}{2}\right)-\frac{1}{2} \log (2) \log ^{2}\left(\frac{1-x}{2}\right)\right)\right]_{+}\right\}(N)+\log (2)\left[S_{1,1,1}(N)-S_{1,1,-1}(N)\right] \\
& -\frac{1}{2}\left[\zeta(2)-\log ^{2}(2)\right] S_{1,1}(N)-\left[\frac{7}{8} \zeta(3)+\frac{1}{2} \zeta(2) \log (2)-\frac{1}{3} \log ^{3}(2)\right] S_{1}(N) \\
& S_{1,1,-1,1}(N)=(-1)^{N} \mathbf{M}\left\{\frac{1}{1+x}\left[\operatorname{Li}_{3}\left(\frac{1+x}{2}\right)-\zeta(2) \log \left(\frac{1+x}{2}\right)\right]\right\}(N)+(-1)^{N+1} \mathbf{M}\left\{\frac{1}{1+x} \frac{1}{2} \log ^{2}\left(\frac{1+x}{2}\right) \log (2)\right\}(N) \\
& -\zeta(3) S_{-1}(N)+\left[\frac{1}{8} \zeta(3)-\frac{1}{2} \zeta(2) \log (2)+\frac{1}{3} \log ^{3}(2)\right] S_{1}(N) \\
& -\frac{1}{2}\left[\zeta(2)-\log ^{2}(2)\right] S_{1,1}(N)-\frac{2}{5} \zeta(2)^{2}+\operatorname{Li}_{4}\left(\frac{1}{2}\right)-\frac{1}{2} \zeta(2) \log ^{2}(2)+\frac{1}{6} \log ^{4}(2)
\end{aligned}
$$




$$
\begin{aligned}
S_{1,1,1,-1}(N)= & (-1)^{N+1} \frac{1}{6} \mathbf{M}\left[\frac{\log ^{3}(1+x)}{1+x}\right](N)+\log (2)\left[S_{1,1,-1}(N)-S_{1,1,1}(N)\right] \\
& +\frac{1}{2} \log ^{2}(2)\left[S_{1,1}(N)-S_{1,-1}(N)\right]-\frac{1}{6} \log ^{3}(2)\left[S_{1}(N)-S_{-1}(N)\right]+\frac{1}{24} \log ^{4}(2) \\
S_{1,1,1,1}(N)= & \frac{1}{6} \mathbf{M}\left[\left(\frac{\log ^{3}(1-x)}{1-x}\right)_{+}\right](N) \\
= & \frac{1}{24}\left[\psi(N+1)+\gamma_{E}\right]^{4}-\frac{1}{4}\left[\psi(N+1)+\gamma_{E}\right]^{2}\left[\psi^{\prime}(N+1)-\zeta(2)\right] \\
& +\frac{1}{3}\left[\psi(N+1)+\gamma_{E}\right]\left[\frac{1}{2} \psi^{\prime \prime}(N+1)+\zeta(3)\right]+\frac{1}{8}\left[\psi^{\prime}(N+1)-\zeta(2)\right]^{2}-\frac{1}{4}\left[\frac{1}{6} \psi^{(3)}(N+1)-\frac{2}{5} \zeta(2)^{2}\right] .
\end{aligned}
$$

The respective combinations of the linear representations of individual harmonic sums may be compared with the algebraic representations of these combinations as given in Sec. V. In this way interesting convolution relations between Nielsen functions may be obtained, which we do not work out further here.

For the above relations extensive checks have been performed by thorough numerical precision test and using computer algebra programs. As a further check we compared the limit

$$
\lim _{N \rightarrow \infty} S_{k_{1}, \ldots, k_{l}}(N)=\sigma_{k_{1}, \ldots, k_{l}}
$$

with the respective multiple $\zeta$ values in the cases when $k_{1}$ $\neq 1$. Analytic results for $\sigma_{k_{1}}, \ldots, k_{l}$ are obtained straightforwardly from the integral Euler-Poincaré [45] integral representation Eqs. (14)-(17) for $N \rightarrow \infty$. Nearly all level-four multiple $\zeta$ values were given by Gastmans and Troost [46]. The missing ones are

$$
\begin{aligned}
\sigma_{-1,-3}= & 2 \operatorname{Li}_{4}\left(\frac{1}{2}\right)-\frac{1}{2} \zeta(4)+\frac{3}{4} \zeta(3) \log (2) \\
& -\frac{1}{2} \zeta(2) \log ^{2}(2)+\frac{1}{12} \log ^{4}(2) \\
\sigma_{-1,+3}= & -\frac{19}{16} \zeta(4)+\frac{3}{4} \zeta(3) \log (2) .
\end{aligned}
$$

In the integrations extensive use has been made of the integrals for Nielsen functions [31,46-48].

\section{ALGEBRAIC RELATIONS BETWEEN HARMONIC SUMS}

The finite harmonic sums are connected by various algebraic relations. We will only consider those among the har- monic sums themselves. ${ }^{7}$ One class of relations is obtained by considering the full set of permutations of an index set and interchanging the order of summation. A second class follows from partial permutations.

\section{A. Complete index permutation}

In the case of two indices the relation was given firstly by Euler [15] for $N \rightarrow \infty$, see also [49-51]. Below we give the generalizations up to four (alternating) indices and consider also relations for structurally more simple harmonic sums beyond transcendentality four. Due to the index symmetry the combinations listed below are polynomials of the simple harmonic sums only, i.e. the Mellin transform to $x$ space of these combinations is a linear combination out of polynomials of Mellin convolutions of the basic functions

$$
\begin{aligned}
& \frac{(-1)^{n}}{\Gamma(n)}\left(\frac{\log ^{n-1}(x)}{x-1}\right)_{+}=\mathbf{M}^{-1}\left[S_{n}(N)\right](x) \\
& \frac{(-1)^{N}}{x+1}-\log (2) \delta(1-x)=\mathbf{M}^{-1}\left[S_{-1}(N)\right](x) \\
& \frac{(-1)^{N+n-1}}{\Gamma(n)}\left(\frac{\log ^{n-1}(x)}{x+1}\right)-\left(1-\frac{1}{2^{n-1}}\right) \zeta(n) \delta(1-x) \\
& =\mathbf{M}^{-1}\left[S_{-n}(N)\right](x), \quad n \in \mathbf{N}, n>1
\end{aligned}
$$

only. As will be shown below, the permutation-symmetric combinations obey also nested representations by lower level harmonic sums, which are not single. By comparing the algebraic relations given below with the linear representations of the previous section one may find convolution relations between Nielsen functions.

\footnotetext{
${ }^{7}$ For the study of relations between infinite harmonic sums the introduction of non-harmonic rest terms which vanish as $N \rightarrow \infty$ may sometimes be useful [18].
} 
The general permutation relation for sums with two indices reads

$$
\begin{aligned}
S_{m, n}+S_{n, m} & =S_{m} S_{n}+S_{\operatorname{sign}\{m\} \operatorname{sign}\{n\}[|m|+|n|]} \\
& \equiv S_{m} S_{n}+S_{m \wedge n}
\end{aligned}
$$

A geometric argument for its derivation is the following. The sums $S_{m, n}$ and $S_{n, m}$ correspond to congruent rectangular triangles which may be supplemented to form the rectangle $S_{m} S_{n}$. Since both triangles overlap on the diagonal of the rectangle $S_{m \wedge n}$ has to be added.

In this way one obtains up to the level of transcendentality four 14 relations of the type (125), which read

$$
\begin{aligned}
S_{1,1} & =\frac{1}{2}\left[S_{1}^{2}+S_{2}\right] \\
S_{1,-1}+S_{-1,1} & =S_{1} S_{-1}+S_{-2} \\
S_{-1,-1} & =\frac{1}{2}\left[S_{-1}^{2}+S_{2}\right] \\
S_{1,2}+S_{2,1} & =S_{1} S_{2}+S_{3} \\
S_{-1,2}+S_{2,-1} & =S_{-1} S_{2}+S_{-3} \\
S_{1,-2}+S_{-2,1} & =S_{1} S_{-2}+S_{-3} \\
S_{-1,-2}+S_{-2,-1} & =S_{-1} S_{-2}+S_{3}
\end{aligned}
$$

$$
\begin{aligned}
S_{2,2} & =\frac{1}{2}\left[S_{2}^{2}+S_{4}\right] \\
S_{2,-2}+S_{-2,2} & =S_{2} S_{-2}+S_{-4} \\
S_{-2,-2} & =\frac{1}{2}\left[S_{-2}^{2}+S_{4}\right] \\
S_{1,3}+S_{3,1} & =S_{1} S_{3}+S_{4} \\
S_{-1,3}+S_{3,-1} & =S_{-1} S_{3}+S_{-4} \\
S_{1,-3}+S_{-3,1} & =S_{1} S_{-3}+S_{-4} \\
S_{-1,-3}+S_{-3,-1} & =S_{-1} S_{-3}+S_{4}
\end{aligned}
$$

Similarly, one obtains for three indices

$$
\sum_{\text {perm }\{l, m, n\}} S_{l, m, n}=S_{l} S_{m} S_{n}+\sum_{\text {inv }} \sum_{\operatorname{perm}\{l, m, n\}} S_{l} S_{m \wedge n}+2 S_{l \wedge m \wedge n},
$$

where "inv perm" denotes the invariant permutations and " 'perm', all permutations. In a geometric interpretation one may identify the sums $S_{l, m, n}$ with the six pyramids of equal volume, ${ }^{8}$ which form the square stone $S_{l} S_{m} S_{n}$. The terms $\sum_{\text {inv perm }\{l, m, n\}} S_{l} S_{m \wedge n}$ account for additional overlapping surfaces in the interior of the square stone and $2 S_{l \wedge m \wedge n}$ for overlapping internal edges. The corresponding relation for infinite sums was derived in Ref. [54]. ${ }^{9}$

Up to transcendentality four 10 relations are obtained:

$$
\begin{aligned}
S_{-1,-1,-1} & =\frac{1}{6} S_{-1}^{3}+\frac{1}{2} S_{-1} S_{2}+\frac{1}{3} S_{-3} \\
& =\frac{1}{3}\left[S_{-1,-1} S_{-1}+S_{-1} S_{2}+S_{-3}\right] \\
S_{-1,-1,1}+S_{-1,1,-1}+S_{1,-1,-1} & =\frac{1}{2}\left[S_{-1}^{2}+S_{2}\right] S_{1}+S_{-1} S_{-2}+S_{3} \\
& =S_{-1,-1} S_{1}+S_{-1} S_{-2}+S_{3} \\
S_{-1,1,1}+S_{1,-1,1}+S_{1,1,-1} & =\frac{1}{2}\left[S_{1}^{2}+S_{2}\right] S_{-1}+S_{1} S_{-2}+S_{-3} \\
= & S_{1,1} S_{-1}+S_{1} S_{-2}+S_{-3}
\end{aligned}
$$

\footnotetext{
${ }^{8}$ Because of the solution [52] of the 3rd Hilbert problem [53] these pyramids are not congruent in general.

${ }^{9}$ For related work see also [55].
} 


$$
\begin{aligned}
& S_{1,1,1}=\frac{1}{6} S_{1}^{3}+\frac{1}{2} S_{1} S_{2}+\frac{1}{3} S_{3} \\
& =\frac{1}{3}\left[S_{1,1} S_{1}+S_{1} S_{2}+S_{3}\right] \\
& S_{2,1,1}+S_{1,2,1}+S_{1,1,2}=\frac{1}{2}\left[S_{1}^{2}+S_{2}\right] S_{2}+S_{1} S_{3}+S_{4} \\
& =S_{1,1} S_{2}+S_{1} S_{3}+S_{4} \\
& S_{2,-1,1}+S_{2,1,-1}+S_{1,2,-1}+S_{1,-1,2}+S_{-1,2,1}+S_{-1,1,2}=S_{1} S_{-1} S_{2}+S_{2} S_{-2}+S_{1} S_{-3}+S_{-1} S_{3}+2 S_{-4} \\
& S_{-2,-1,1}+S_{-2,1,-1}+S_{1,-2,-1}+S_{1,-1,-2}+S_{-1,-2,1}+S_{-1,1,-2}=S_{1} S_{-1} S_{-2}+S_{-2}^{2}+S_{1} S_{3}+S_{-1} S_{-3}+2 S_{4} \\
& S_{2,-1,-1}+S_{-1,2,-1}+S_{-1,-1,2}=\frac{1}{2}\left[S_{-1}^{2}+S_{2}\right] S_{2}+S_{-1} S_{-3}+S_{4} \\
& =S_{-1,-1} S_{2}+S_{-1} S_{-3}+S_{4} \\
& S_{-2,1,1}+S_{1,-2,1}+S_{1,1,-2}=\frac{1}{2}\left[S_{1}^{2}+S_{2}\right] S_{-2}+S_{-1} S_{-3}+S_{-4} \\
& =S_{1,1} S_{-2}+S_{-1} S_{-3}+S_{-4} \\
& S_{-2,-1,-1}+S_{-1,-2,-1}+S_{-1,-1,-2}=\frac{1}{2}\left[S_{-1}^{2}+S_{2}\right] S_{-2}+S_{-1} S_{3}+S_{-4} \\
& =S_{-1,-1} S_{-2}+S_{-1} S_{3}+S_{-4} .
\end{aligned}
$$

Wherever possible we gave a second representation collecting part of the terms into double sums which were given before.

Finally, the relations for sums with four indices read

$$
\sum_{\operatorname{perm}\{k, l, m, n\}} S_{k, l, m, n}=S_{k} S_{l} S_{m} S_{n}+\sum_{\text {inv } \operatorname{perm}\{k, l, m, n\}} S_{k} S_{l} S_{m \wedge n}+\sum_{\text {inv perm }\{k, l, m, n\}} S_{k \wedge l} S_{m \wedge n}+2 \sum_{\text {inv perm }\{k, l, m, n\}} S_{k} S_{l \wedge m \wedge n}+6 S_{k \wedge l \wedge m \wedge n}
$$

One obtains 5 other relations up to level four,

$$
\begin{aligned}
& S_{-1,-1,-1,-1}=\frac{1}{4} S_{4}+\frac{1}{8} S_{2}^{2}+\frac{1}{3} S_{-3} S_{-1}+\frac{1}{4} S_{2} S_{-1}^{2}+\frac{1}{24} S_{-1}^{4} \\
&=\frac{1}{4}\left[S_{-1,-1,-1} S_{-1}+S_{-1,-1} S_{2}+S_{-1} S_{-3}+S_{4}\right] \\
& S_{1,-1,-1,-1}+S_{-1,1,-1,-1}+S_{-1,-1,1,-1}+S_{-1,-1,-1,1}=\frac{1}{6} S_{1}^{3} S_{-1}+\frac{1}{3} S_{-1} S_{3}+S_{1} S_{-3}+S_{-4}+\frac{1}{2}\left[S_{1}^{2} S_{-2}+S_{1} S_{-1} S_{2}+S_{2} S_{-2}\right] \\
&= S_{1,1,1} S_{-1}+S_{1,1} S_{-2}+S_{1} S_{-3}+S_{-4} \\
& 014018-16
\end{aligned}
$$




$$
\begin{aligned}
S_{-1,-1,1,1}+ & S_{-1,1,-1,1}+S_{-1,1,1,-1}+S_{1,1,-1,-1}+S_{1,-1,1,-1}+S_{1,-1,-1,1} \\
= & S_{-1}^{2} S_{1}^{2}+S_{-1}^{2} S_{2}+4 S_{-1} S_{1} S_{-2}+S_{2} S_{1}^{2}+S_{2}^{2}+2 S_{-2}^{2}+4 S_{-1} S_{-3}+4 S_{1} S_{3}+S_{4} \\
= & 4\left[S_{1,1} S_{-1,-1}+S_{-2,-2}+S_{-1,-3}+S_{-3,-1}+S_{1,3}+S_{3,1}-S_{4}\right] \\
S_{-1,1,1,1}+S_{1,-1,1,1}+S_{1,1,-1,1}+S_{1,1,1,-1}= & \frac{1}{6} S_{-1}^{3} S_{1}+\frac{1}{3} S_{-3} S_{1}+S_{-1} S_{3}+S_{-4} \\
& +\frac{1}{2}\left[S_{-1} S_{1} S_{2}+S_{-1}^{2} S_{-2}+S_{-2} S_{2}\right] \\
= & S_{-1,-1,-1} S_{1}+S_{-1,-1} S_{-2}+S_{-1} S_{3}+S_{-4} \\
S_{1,1,1,1}= & \frac{1}{4} S_{4}+\frac{1}{8} S_{2}^{2}+\frac{1}{3} S_{3} S_{1}+\frac{1}{4} S_{2} S_{1}^{2}+\frac{1}{24} S_{1}^{4} \\
= & \frac{1}{4}\left[S_{1,1,1} S_{1}+S_{1,1} S_{2}+S_{1} S_{3}+S_{4}\right] .
\end{aligned}
$$

More relations are given in Ref. [20].

The sums of the type $S_{-1,-1, \ldots,-1}$ can be evaluated in compact form at arbitrary depth:

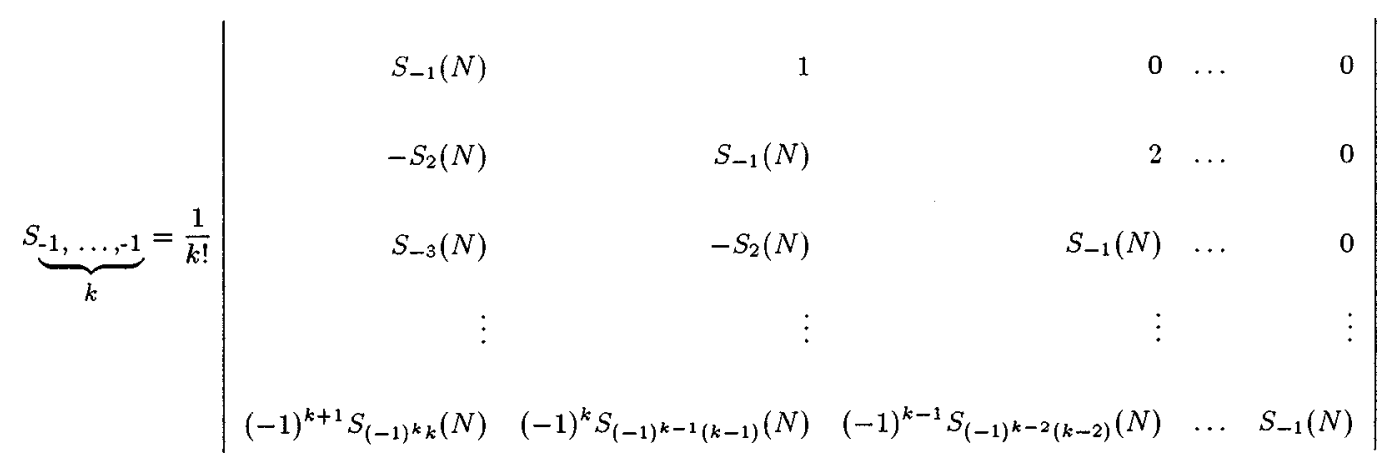

Similarly the corresponding expressions for $S_{1,1, \ldots, 1}$ are

$$
\underbrace{S_{1, \ldots, 1}}_{k}=\frac{1}{k !}\left|\begin{array}{rrrrrr}
S_{1}(N) & 1 & 0 & 0 & \ldots & 0 \\
-S_{2}(N) & S_{1}(N) & 2 & 0 & \ldots & 0 \\
S_{3}(N) & -S_{2}(N) & S_{1}(N) & 3 & \ldots & 0 \\
\vdots & \vdots & \vdots & \vdots & & \vdots \\
(-1)^{k+1} S_{k}(N) & (-1)^{k} S_{k-1}(N) & (-1)^{k-1} S_{k-2}(N) & (-1)^{k-2} S_{k-3}(N) & \ldots & S_{1}(N)
\end{array}\right|
$$


which are given by

$$
\begin{aligned}
& S_{5}^{S_{1, \ldots,-1}}=\frac{1}{120} S_{-1}^{5}+\frac{1}{12} S_{2} S_{-1}^{3}+\frac{1}{6} S_{-3} S_{-1}^{2}+\frac{1}{4} S_{4} S_{-1}+\frac{1}{8} S_{-1} S_{2}^{2}+\frac{1}{6} S_{2} S_{-3} \\
& +\frac{1}{5} S_{-5} \\
& S_{6}^{S_{1, \ldots,-1}}=\frac{1}{720} S_{-1}^{6}+\frac{1}{48} S_{2} S_{-1}^{4}+\frac{1}{18} S_{-3} S_{-1}^{3}+\frac{1}{8} S_{4} S_{-1}^{2}+\frac{1}{5} S_{-5} S_{-1}+\frac{1}{16} S_{-1}^{2} S_{2}^{2} \\
& +\frac{1}{6} S_{-1} S_{2} S_{-3}+\frac{1}{48} S_{2}^{3}+\frac{1}{8} S_{2} S_{4}+\frac{1}{18} S_{-3}^{2}+\frac{1}{6} S_{6} \\
& S_{7}^{-1, \ldots,-1}=\frac{1}{5040} S_{-1}^{7}+\frac{1}{240} S_{-1}^{5} S_{2}+\frac{1}{72} S_{-1}^{4} S_{-3}+\frac{1}{24} S_{-1}^{3} S_{4}+\frac{1}{10} S_{-1}^{2} S_{-5}+\frac{1}{6} S_{-1} S_{6} \\
& +\frac{1}{10} S_{2} S_{-5}+\frac{1}{24} S_{2}^{2} S_{-3}+\frac{1}{48} S_{2}^{2} S_{-1}^{3}+\frac{1}{48} S_{2}^{3} S_{-1}+\frac{1}{12} S_{-3} S_{4}+\frac{1}{18} S_{-3}^{2} S_{-1} \\
& +\frac{1}{8} S_{-1} S_{2} S_{4}+\frac{1}{12} S_{-1}^{2} S_{2} S_{-3}+\frac{1}{7} S_{-7} \\
& S_{8}^{S_{1, \ldots,-1}}=\frac{1}{40320} S_{-1}^{8}+\frac{1}{1440} S_{-1}^{6} S_{2}+\frac{1}{360} S_{-1}^{5} S_{-3}+\frac{1}{96} S_{-1}^{4} S_{4}+\frac{1}{30} S_{-1}^{3} S_{-5} \\
& +\frac{1}{12} S_{-1}^{2} S_{6}+\frac{1}{7} S_{-1} S_{-7}+\frac{1}{36} S_{-1}^{3} S_{2} S_{-3}+\frac{1}{16} S_{-1}^{2} S_{2} S_{4}+\frac{1}{24} S_{-1} S_{2}^{2} S_{-3} \\
& +\frac{1}{12} S_{-1} S_{-3} S_{4}+\frac{1}{96} S_{-1}^{2} S_{2}^{3}+\frac{1}{36} S_{-1}^{2} S_{-3}^{2}+\frac{1}{384} S_{2}^{4}+\frac{1}{192} S_{2}^{2} S_{-1}^{4}+\frac{1}{32} S_{2}^{2} S_{4} \\
& +\frac{1}{36} S_{2} S_{-3}^{2}+\frac{1}{12} S_{2} S_{6}+\frac{1}{10} S_{2} S_{-1} S_{-5}+\frac{1}{15} S_{-3} S_{-5}+\frac{1}{32} S_{4}^{2}+\frac{1}{8} S_{8}
\end{aligned}
$$

in explicit form beyond level 4. The corresponding relations for

$$
S_{n}^{1, \ldots, 1}
$$

are obtained by substituting the indices $-k \rightarrow k$ in Eqs. (159)-(162). Note the similarity to the corresponding sums of positive integer powers studied by F. Faà di Bruno $[57,58] .^{10}$

One may evaluate these sums also using the recursion relations

$$
\begin{aligned}
S_{\underbrace{1, \ldots,-1}} & =\frac{1}{k} \sum_{l=1}^{k} S_{(-1)^{l}|l|} S_{S_{k-l}^{1, \ldots,-1}} \\
S_{k}^{S_{1, \ldots, 1}} & =\frac{1}{k} \sum_{l=1}^{k} S_{l} S_{\underbrace{1, \ldots, 1}_{k-1}} .
\end{aligned}
$$

Sums of this type were studied already long ago $[59,60]$. A relation to derivatives of the $\Gamma$ function was given in [50]. Recently a generating function for the sums

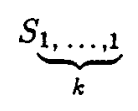

has been derived [35]

$$
(-1)^{N} x B(N+1, x-N)=1+\sum_{l=1}^{\infty} x^{l} S_{\underbrace{1, \ldots, 1}_{l}}(N) .
$$

Finally we would like to mention a relation (cf. [61]) similar to those for

\footnotetext{
${ }^{10}$ For historical aspects see also [56].
}

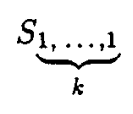


relating the Stirling numbers of the first kind to the finite harmonic sums of positive index,

$$
\left[\begin{array}{l}
n \\
m
\end{array}\right]=(n-1)\left[\begin{array}{c}
n-1 \\
m
\end{array}\right]+\left[\begin{array}{c}
n-1 \\
m-1
\end{array}\right]=\frac{\Gamma(n)}{\Gamma(m)} w(n, m-1),
$$

with

$$
\begin{aligned}
w(n, 0) & =1 \\
w(n, m) & =\sum_{k=0}^{m-1}(1-m)_{k} S_{k+1}(n-1) w(n, m-1-k)
\end{aligned}
$$

and $(r)_{k}=\Pi_{l=1}^{k}(r+k-1)$ the Pochhammer-Barnes symbol.

The permutation relations lead to a first reduction of the number of direct Mellin transforms (39)-(118) and relate the transcendental functions occurring as arguments of the transforms in the representations of Sec. IV.

\section{B. Partial permutations and triple harmonic sums}

Aside of the permutation-invariant sum of harmonic sums over a given index set one can consider equivalent decompositions of more symmetric sums to obtain further algebraic relations. For triple harmonic sums one may study the expression

$$
T_{a, b, c}(N)=\sum_{k=1}^{N} \frac{1}{k^{a}}\left[\sum_{l=1}^{k} \frac{1}{l^{b}}\right]\left[\sum_{m=1}^{k} \frac{1}{m^{b}}\right] .
$$

It was shown by Borwein and Girgensohn [18] for nonalternating finite sums, that $T_{a, b, c}(N)^{11}$ may have four representations in terms of triple harmonic sums. Earlier similar relations were derived by Markett [62] for infinite nonalternating sums. We generalize the relations allowing also for alternating sums. These relations read

$$
\begin{aligned}
& T=S_{a, b, c}+S_{a, c, b}-S_{a \wedge b, c}-S_{a \wedge c, b}-S_{a, b \wedge c}+S_{a \wedge b \wedge c} \\
& T=S_{c} S_{a, b}-S_{c, a, b}+S_{c, a \wedge b}-S_{c} S_{a \wedge b} \\
& T=S_{b} S_{a, c}-S_{b, a, c}+S_{b, a \wedge c}-S_{b} S_{a \wedge c} \\
& T=S_{b, c, a}+S_{c, b, a}-S_{b \wedge c, a}-S_{c} S_{b, a}+S_{b} S_{a, c}-S_{b} S_{a \wedge c} .
\end{aligned}
$$

They result into three relations between the harmonic sums for $a, b$ and $c$ being different and into two relations for two of the variables being equal. The global permutation relation Eq. (140) is a consequence of Eqs. (169)-(172).

\footnotetext{
${ }^{11}$ In Ref. [18] relations between the sums $\eta_{N}(k, l, m)=S_{k, l, m}(N)$ $-S_{k+l, m}(N)-S_{k, l+m}(N)+S_{k+l+m}(N)$ were discussed. We rewrite them in terms of the sums $S_{k_{1}}, \ldots, k_{r}$ used throughout the present paper.
}

Let us finally write down the relations for the cases discussed in the present paper explicitly. The basis elements were choosen already according to the results of the previous section, taking those sums as basis elements which have the most simple functional structure with respect to the argument of the Mellin transform. We have no algebraic argument to predict this structure, which had to be found by explicit analytic integration. To choose the most simple structures as basis elements has the advantage that the analytic representations of the Mellin transforms for complex values of $N$, cf. [43], becomes as simple as possible. For the level-3 sums the following relations hold:

$$
S_{-1,-1,1}=\frac{1}{2}\left(-S_{-1,1,-1}+S_{-1} S_{-1,1}+S_{-1,-2}+S_{2,1}\right)
$$

$$
\begin{aligned}
S_{1,-1,-1}= & \frac{1}{2}\left(-S_{-1,1,-1}+S_{-1} S_{1,-1}-S_{2,1}-S_{-1,-2}\right. \\
& \left.+S_{1} S_{2}+S_{-1} S_{-2}+2 S_{3}\right) \\
S_{-1,1,1}= & S_{1,1,-1}-S_{1} S_{1,-1}+S_{-2,1}+S_{-1,2} \\
& +\frac{1}{2}\left(S_{1}^{2} S_{-1}-S_{-1} S_{2}\right)-S_{-3} \\
S_{1,-1,1}= & -2 S_{1,1,-1}+S_{1} S_{1,-1}-S_{-2,1}-S_{-1,2}+S_{1} S_{-2} \\
& +S_{-1} S_{2}+2 S_{-3} .
\end{aligned}
$$

The relations for the threefold level-4 sums are :

$$
\begin{aligned}
& S_{1,2,1}=-2 S_{2,1,1}+S_{3,1}+S_{1} S_{2,1}+S_{2,2} \\
& S_{1,1,2}=S_{2,1,1}+\frac{1}{2}\left[S_{1}\left(S_{1,2}-S_{2,1}\right)+S_{1,3}-S_{3,1}\right]
\end{aligned}
$$

$$
\begin{aligned}
S_{1,-2,1}= & -2 S_{-2,1,1}+S_{-3,1}+S_{1} S_{-2,1}+S_{-2,2} \\
S_{1,1,-2}= & S_{-2,1,1}+S_{-2} S_{2}-S_{-2,2}-S_{-2} S_{1,1} \\
& +S_{1} S_{1,-2}+S_{1,-3}-S_{1} S_{-3} \\
S_{-1,-1,2}= & \frac{1}{2}\left(S_{-1} S_{-1,2}+S_{-1,-3}+S_{2,2}-S_{-1,2,-1}\right)
\end{aligned}
$$

$$
\begin{aligned}
S_{2,-1,-1}= & \frac{1}{2}\left(-S_{-1,2,-1}+S_{-3,-1}\right. \\
& \left.+S_{-1} S_{2,-1}+S_{2,2}\right)
\end{aligned}
$$

$$
\begin{aligned}
S_{-1,-1,-2}= & \frac{1}{2}\left(-S_{-1,-2,-1}+S_{2,-2}+S_{-1} S_{-1,-2}\right. \\
& \left.+S_{-1,3}\right)
\end{aligned}
$$




$$
\begin{aligned}
S_{-2,-1,-1}= & \frac{1}{2}\left(-S_{-1,-2,-1}+S_{-2,2}+S_{3,-1}\right. \\
& \left.+S_{-1} S_{-2,-1}\right) \\
S_{-2,-1,1}= & -S_{1,-2,-1}-S_{-2,1,-1}+S_{3,1}+S_{-3,-1}+S_{4} \\
& +S_{1} S_{-2,-1}+S_{1,3}-S_{1} S_{3}+S_{-2,-2} \quad(185) \\
S_{1,-1,-2}= & -S_{-1,-2,1}-S_{-1,1,-2}+S_{-1,-3}+S_{-2,-2} \\
& +S_{1} S_{-1,-2} \\
S_{1,-2,-1}= & S_{-1,-2,1}-S_{-1} S_{-2,1}-S_{-1,-3}+S_{-1} S_{-3} \\
& +S_{1} S_{-2,-1}+S_{1,3}-S_{1} S_{3} \\
S_{2,-1,1}= & -S_{1,2,-1}-S_{2,1,-1}+S_{3,-1}+S_{2}\left(S_{-1,1}\right.
\end{aligned}
$$

$$
\begin{aligned}
& \left.+S_{1,-1}\right)+S_{2,-2}-S_{2} S_{-2}-S_{1} S_{-1,2} \\
& +S_{1} S_{-3} \\
S_{1,-1,2}= & -S_{1,2,-1}-S_{2,1,-1}+S_{1,-3}+S_{3,-1} \\
& +S_{2} S_{1,-1} \\
S_{-1,1,2}= & S_{1,2,-1}+S_{2,1,-1}-S_{-1,2,1}+S_{-2,2}+S_{-3,1} \\
& -S_{-4}+S_{-1,3}-S_{3,-1}-S_{2} S_{1,-1} \\
& +S_{1} S_{-1,2}-S_{1} S_{-3} .
\end{aligned}
$$

Using the algebraic relations given above one may show that the linear representations up to threefold sums given in Sec. IV can be expressed by the single harmonic sums $S_{ \pm k}(N)$ and the Mellin transforms of the functions

$$
\begin{array}{cccc}
\frac{\log (1+x)}{x+1} & \frac{\log ^{2}(1+x)-\log ^{2}(2)}{x-1} & \frac{\log ^{2}(1+x)}{x+1} & \frac{\operatorname{Li}_{2}(x)}{x+1} \\
\frac{\mathrm{Li}_{2}(x)-\zeta(2)}{x-1} & \frac{\mathrm{Li}_{2}(-x)}{x+1} & \frac{\mathrm{Li}_{2}(-x)+\zeta(2) / 2}{x-1} & \frac{\log (x) \mathrm{Li}_{2}(x)}{x+1} \\
\frac{\log (x) \mathrm{Li}_{2}(x)}{x-1} & \frac{\mathrm{Li}_{3}(x)}{x+1} & \frac{\mathrm{Li}_{3}(x)-\zeta(3)}{x-1} & \frac{\mathrm{Li}_{3}(-x)}{x+1} \\
\frac{\mathrm{Li}_{3}(-x)-3 \zeta(3) / 4}{x-1} & \frac{\mathrm{S}_{1,2}(x)}{x+1} & \frac{\mathrm{S}_{1,2}(x)-\zeta(3)}{x-1} & \frac{\mathrm{S}_{1,2}(-x)-\zeta(3) / 8}{x-1} \\
\frac{\mathrm{S}_{1,2}(-x)}{x+1} & \frac{\mathrm{S}_{1,2}\left(x^{2}\right)}{x+1} & \frac{\mathrm{S}_{1,2}\left(x^{2}\right)-\zeta(3)}{x-1} & \log (1-x) \frac{\mathrm{Li}_{2}(-x)}{x+1} \\
\frac{\log (1+x)-\log (2)}{x-1} \mathrm{Li}_{2}(x) & \frac{\log (1+x)-\log (2)}{x-1} \mathrm{Li}_{2}(-x) & \frac{\log (1-x) \operatorname{Li}(x)}{1+x} & \frac{\log (1+x)}{1+x} \operatorname{Li}_{2}(x) .
\end{array}
$$

According to Carlson's theorem [63] the analytic continuation of Mellin transforms to complex numbers of $N$ can be performed uniquely. The corresponding expressions for the Mellin transforms of the functions in Eq. (191) are given in Ref. [43].

The Mellin transform of complicated functions as $F_{1}(x)$, Eq. (38), or Nielsen functions with more complicated arguments as $(1 \pm x) / 2$, which occur in the triple sums with the indices \pm 1 , can be represented by polynomials of the Mellin transforms of the functions listed above.

\section{CONCLUSIONS}

The Mellin transforms of the Nielsen functions which emerge in the splitting functions and Wilson coefficients in massless QED and QCD up to two-loop order were calculated for all the contributing functions $f_{i}(x)$. They can be expressed by finite harmonic sums up to level four. The mathematical properties of Mellin transforms of this class can be understood easiest from the point of view of the alternating and non-alternating finite harmonic sums. These are polynomials over a substet of harmonic sums, which are related to Mellin transforms of Nielsen functions of relatively simple functional or argument structure. The latter properties could only be revealed by the explicit calculation of all finite harmonic sums up to level four in the linear representation. Comparing the linear representation with the representations induced by the algebraic relations one may obtain a class of linear combinations of convolution relations between Nielsen functions. The fourfold level-four sums are 
found to contribute to the splitting functions and Wilson coefficients up to two loop order only in their simplest variants, which can be represented algebraically in terms of lower level sums. They are, however, expected to contribute to the quantities beyond the two-loop level. All remaining harmonic sums can be constructed out of about 20 basic Mellin transforms and the known representations which are expressible in terms of the function $\psi^{k}(z)$. The splitting functions and coefficient functions depend usually only on a subset of these harmonic sums requiring even less basic functions for the representation of their Mellin transforms.

\section{ACKNOWLEDGMENTS}

We would like to thank W. van Neerven, V. Ravindran and J. Vermaseren for useful discussions. This work was supported in part by EU contract FMRX-CT98-0194 (DG12MIHT).

\section{APPENDIX: MELLIN TRANSFORMS}

We list here the full set of functions $f_{i}(x)$ and their Mellin transforms $\mathbf{M}\left[f_{i}(x)\right]=\int_{0}^{1} d x x^{N-1} f_{i}(x)$ in terms of the linear representations derived in Sec. IV:

$$
\begin{aligned}
& \mathbf{M}[\delta(1-z)](N)=1, \\
& \mathbf{M}\left(z^{r}\right)(N)=\frac{1}{N+r} \\
& \mathbf{M}\left[\left(\frac{1}{1-z}\right)_{+}\right](N)=-S_{1}(N-1), \\
& \mathbf{M}\left(\frac{1}{1+z}\right)(N)=(-1)^{N-1}\left[\log (2)-S_{1}(N-1)\right]+\frac{1+(-1)^{N-1}}{2} S_{1}\left(\frac{N-1}{2}\right)-\frac{1-(-1)^{N-1}}{2} S_{1}\left(\frac{N-2}{2}\right), \\
& \mathbf{M}\left[z^{r} \log ^{n}(z)\right](N)=\frac{(-1)^{n}}{(N+r)^{n+1}} \Gamma(n+1), \\
& \mathbf{M}\left[z^{r} \log (1-z)\right](N)=-\frac{S_{1}(N+r)}{N+r}, \\
& \mathbf{M}\left[z^{r} \log ^{2}(1-z)\right](N)=\frac{S_{1}^{2}(N+r)+S_{2}(N+r)}{N+r}, \\
& \mathbf{M}\left[z^{r} \log ^{3}(1-z)\right](N)=-\frac{S_{1}^{3}(N+r)+3 S_{1}(N+r) S_{2}(N+r)+2 S_{3}(N+r)}{N+r}, \\
& \mathbf{M}\left[\left(\frac{\log (1-z)}{1-z}\right)_{+}\right](N)=\frac{1}{2} S_{1}^{2}(N-1)+\frac{1}{2} S_{2}(N-1), \\
& \mathbf{M}\left[\left(\frac{\log ^{2}(1-z)}{1-z}\right)_{+}\right](N)=-\left[\frac{1}{3} S_{1}^{3}(N-1)+S_{1}(N-1) S_{2}(N-1)+\frac{2}{3} S_{3}(N-1)\right], \\
& \mathbf{M}\left[\left(\frac{\log ^{3}(1-z)}{1-z}\right)_{+}\right](N)=\frac{1}{4} S_{1}^{4}(N-1)+\frac{3}{2} S_{1}^{2}(N-1) S_{2}(N-1) \\
& +\frac{3}{4} S_{2}^{2}(N-1)+2 S_{1}(N-1) S_{3}(N-1)+\frac{3}{2} S_{4}(N-1), \\
& \mathbf{M}\left(\frac{\log ^{n}(z)}{1-z}\right)(N)=(-1)^{n+1} \Gamma(n+1)\left[S_{n+1}(N-1)-\zeta(n+1)\right]
\end{aligned}
$$




$$
\begin{aligned}
& \mathbf{M}\left[z^{r} \log (1+z)\right](N)=\frac{(-1)^{N+r-1}}{N+r}\left[-S_{1}(N+r)+\frac{1+(-1)^{N+r-1}}{2} \times S_{1}\left(\frac{N+r-1}{2}\right)+\frac{1-(-1)^{N+r-1}}{2} S_{1}\left(\frac{N+r}{2}\right)\right] \\
& +\left[1+(-1)^{N+r+1}\right] \frac{\log (2)}{N+r} \\
& \mathbf{M}\left[\log ^{2}(1+z)\right](N)=\frac{1}{N}\left\{\log ^{2} 2-2 \mathbf{M}\left[\frac{\log (1+x)}{1+x}\right](N+1)\right\} \\
& \mathbf{M}\left(\frac{\log ^{n}(z)}{1+z}\right)(N)=(-1)^{n} \Gamma(n+1)\left\{(-1)^{N}\left[S_{n+1}(N-1)-\zeta(n+1)\right]+\frac{1+(-1)^{N-1}}{2^{n+1}}\left[S_{n+1}\left(\frac{N-1}{2}\right)-\zeta(n+1)\right]\right. \\
& \left.-\frac{1-(-1)^{N-1}}{2^{n+1}}\left[S_{n+1}\left(\frac{N-2}{2}\right)-\zeta(n+1)\right]\right\} \\
& \mathbf{M}\left(\frac{\log (1-z)}{1+z}\right)(N)=(-1)^{N}\left[S_{-1,1}(N-1)+\frac{\zeta(2)}{2}-\frac{\log ^{2} 2}{2}\right] \\
& \mathbf{M}\left(\frac{\log (1+z)}{1+z}\right)(N)=(-1)^{N-1}\left[S_{-1,1}(N-1)+S_{1}^{2}(N-1)+S_{2}(N-1)-2 S_{1}(N-1) \log 2+\frac{\log ^{2} 2}{2}\right]-\frac{1+(-1)^{N-1}}{2} \\
& \times\left[S_{1}(N-1) S_{1}\left(\frac{N-1}{2}\right)-S_{1}\left(\frac{N-1}{2}\right) \log 2+\frac{1}{2} S_{2}\left(\frac{N-1}{2}\right)\right]+\frac{1-(-1)^{N-1}}{2}\left[S_{1}(N-1) S_{1}\left(\frac{N-2}{2}\right)\right. \\
& \left.-S_{1}\left(\frac{N-2}{2}\right) \log 2+\frac{1}{2} S_{2}\left(\frac{N-2}{2}\right)\right] \\
& \mathbf{M}\left[z^{r} \log (z) \log (1-z)\right](N)=\frac{S_{1}(N+r)}{(N+r)^{2}}+\frac{1}{N+r}\left[S_{2}(N+r)-\zeta(2)\right], \\
& \mathbf{M}\left[z^{r} \log ^{2}(z) \log (1-z)\right](N)=\frac{2}{N+r}\left[\zeta(3)+\frac{\zeta(2)}{N+r}-\frac{S_{1}(N+r)}{(N+r)^{2}}-\frac{S_{2}(N+r)}{N+r}-S_{3}(N+r)\right], \\
& \mathbf{M}\left(\frac{1}{1-z} \log (z) \log (1-z)\right)(N)=\zeta(3)+\zeta(2) S_{1}(N-1)-S_{1}(N-1) S_{2}(N-1)-S_{3}(N-1)
\end{aligned}
$$

$$
\begin{aligned}
\mathbf{M}\left[z^{r} \log (z) \log (1+z)\right](N)= & (-1)^{N+r} \frac{\zeta(2)}{2(N+r)}-\frac{1+(-1)^{N+r-1}}{(N+r)^{2}} \log (2)+\frac{(-1)^{N+r}}{N+r} \\
& \times\left[-S_{2}(N+r)+\frac{1+(-1)^{N+r-1}}{4} S_{2}\left(\frac{N+r-1}{2}\right)+\frac{1-(-1)^{N+r-1}}{4} S_{2}\left(\frac{N+r}{2}\right)\right] \\
& +\frac{(-1)^{N+r}}{(N+r)^{2}}\left[-S_{1}(N+r)+\frac{1+(-1)^{N+r-1}}{2} S_{1}\left(\frac{N+r-1}{2}\right)+\frac{1-(-1)^{N+r-1}}{2} S_{1}\left(\frac{N+r}{2}\right)\right],
\end{aligned}
$$




$$
\begin{aligned}
& \mathbf{M}\left(\frac{1}{1+z} \log (z) \log (1+z)\right)(N)=(-1)^{N-1}\left[\frac{\zeta(2)}{2} S_{1}(N-1)+2 S_{2}(N-1) \log 2-2 S_{1}(N-1) S_{2}(N-1)-S_{-2,1}(N-1)\right. \\
& \left.-S_{-1,2}(N-1)-2 S_{3}(N-1)-\frac{\zeta(3)}{8}\right]+\frac{1+(-1)^{N-1}}{4}\left[S_{1}(N-1) S_{2}\left(\frac{N-1}{2}\right)\right. \\
& \left.+2 S_{1}\left(\frac{N-1}{2}\right) S_{2}(N-1)-S_{2}\left(\frac{N-1}{2}\right) \log 2+S_{3}\left(\frac{N-1}{2}\right)\right]-\frac{1-(-1)^{N-1}}{4} \\
& \times\left[S_{1}(N-1) S_{2}\left(\frac{N-2}{2}\right)+2 S_{1}\left(\frac{N-2}{2}\right) S_{2}(N-1)-S_{2}\left(\frac{N-2}{2}\right) \log 2+S_{3}\left(\frac{N-2}{2}\right)\right], \\
& \mathbf{M}\left[z^{r} \log (z) \log ^{2}(1-z)\right](N)=\frac{2}{N+r}\left\{\zeta(3)+\zeta(2) S_{1}(N+r)-\frac{1}{2(N+r)}\left[S_{1}^{2}(N+r)+S_{2}(N+r)\right]\right. \\
& \left.-S_{1}(N+r) S_{2}(N+r)-S_{3}(N+r)\right\}, \\
& \mathbf{M}\left(\frac{1}{1-z} \log (z) \log ^{2}(1-z)\right)(N)=S_{2}^{2}(N-1)-\zeta(2) S_{2}(N-1)+2 S_{4}(N-1)+S_{1}^{2}(N-1)\left[S_{2}(N-1)-\zeta(2)\right] \\
& +2 S_{1}(N-1)\left[S_{3}(N-1)-\zeta(3)\right]-\frac{4}{5} \zeta(2)^{2}, \\
& \mathbf{M}\left(\frac{1}{1-z} \log ^{2}(z) \log (1-z)\right)(N)=-2 \zeta(3) S_{1}(N-1)+2 S_{1}(N-1) S_{3}(N-1)-2 \zeta(2) S_{2}(N-1)+S_{2}^{2}(N-1)+3 S_{4}(N-1) \\
& -\frac{\zeta(2)^{2}}{5} \\
& \mathbf{M}\left(\frac{1}{1+z} \log ^{2}(z) \log (1-z)\right)(N)=2(-1)^{N}\left[S_{-3,1}(N-1)+S_{-1,3}(N-1)+S_{-2,2}(N-1)-\zeta(2) S_{-2}(N-1)-\zeta(3) S_{-1}(N-1)\right. \\
& \left.+2 \mathrm{Li}_{4}\left(\frac{1}{2}\right)-\frac{1}{5} \zeta^{2}(2)-\frac{1}{2} \zeta(2) \log ^{2} 2+\frac{1}{12} \log ^{4} 2\right] \\
& \mathbf{M}\left(\frac{1}{1+z} \log (z) \log ^{2}(1-z)\right)(N)=2(-1)^{N}\left[S_{-2,1,1}(N-1)+S_{-1,1,2}(N-1)+S_{-1,2,1}(N-1)-\zeta(2) S_{-1,1}(N-1)\right. \\
& \left.-\zeta(3) S_{-1}(N-1)+3 \operatorname{Li}_{4}\left(\frac{1}{2}\right)-\frac{11}{20} \zeta^{2}(2)+\frac{1}{8} \log ^{4} 2\right], \\
& \mathbf{M}\left[z^{r} \log ^{2} z \log (1+z)\right](N)=2 \frac{(-1)^{N+r}}{N+r}\left[\frac{S_{1}(N+r)}{(N+r)^{2}}+\frac{S_{2}(N+r)}{N+r}+S_{3}(N+r)-\frac{\zeta(2)}{2(N+r)}-\frac{3 \zeta(3)}{4}\right] \\
& +\frac{1+(-1)^{N+r-1}}{2(N+r)}\left[\frac{2}{(N+r)^{2}} S_{1}\left(\frac{N+r-1}{2}\right)+\frac{1}{N+r} S_{2}\left(\frac{N+r-1}{2}\right)+\frac{1}{2} S_{3}\left(\frac{N+r-1}{2}\right)+\frac{4 \log 2}{(N+r)^{2}}\right] \\
& -\frac{1-(-1)^{N+r-1}}{2(N+r)}\left[\frac{2}{(N+r)^{2}} S_{1}\left(\frac{N+r}{2}\right)+\frac{1}{N+r} S_{2}\left(\frac{N+r}{2}\right)+\frac{1}{2} S_{3}\left(\frac{N+r}{2}\right)\right], \\
& \mathbf{M}\left(\frac{1}{1+z} \log ^{2} z \log (1+z)\right)(N)=(-1)^{N}\left[2 S_{3,-1}(N-1)+2 S_{1,-3}(N-1)+2 S_{2,-2}(N-1)+\zeta(2) S_{2}(N-1)\right. \\
& +\frac{3}{2} \zeta(3) S_{1}(N-1)+2 S_{3}(N-1) \log 2-2 S_{-3}(N-1) \log 2-4 \operatorname{Li}_{4}\left(\frac{1}{2}\right)+\frac{3}{2} \zeta^{2}(2) \\
& \left.-\frac{7}{2} \zeta(3) \log 2+\zeta(2) \log ^{2} 2-\frac{1}{6} \log ^{4} 2\right],
\end{aligned}
$$




$$
\begin{aligned}
\mathbf{M}\left(\frac{1}{1+z} \log z \log ^{2}(1+z)\right)(N)= & 2(-1)^{N}\left[S_{1,1,-2}(N-1)+S_{1,2,-1}(N-1)+S_{2,1,-1}(N-1)-S_{1,-2}(N-1) \log 2\right. \\
& -S_{2,-1}(N-1) \log 2+S_{1}(N-1) S_{2}(N-1) \log 2+\frac{1}{4} \zeta(2) S_{1}^{2}(N-1)+S_{3}(N-1) \log 2 \\
& +\frac{1}{4} \zeta(2) S_{2}(N-1)-\frac{1}{2} S_{2}(N-1) \log ^{2} 2+\frac{1}{2} S_{-2}(N-1) \log ^{2} 2-\frac{1}{8} \zeta(3) S_{1}(N-1)-\mathrm{Li}_{4}\left(\frac{1}{2}\right) \\
& \left.+\frac{2}{5} \zeta^{2}(2)-\frac{7}{8} \zeta(3) \log 2+\frac{1}{4} \zeta(2) \log ^{2} 2-\frac{1}{24} \log ^{4} 2\right]
\end{aligned}
$$

$$
\begin{aligned}
\mathbf{M}\left[z^{r} \log z \log ^{2}(1+z)\right](N)= & -\frac{2}{N+r}\left\{( - 1 ) ^ { N + r } \left[\frac{\zeta(2)}{2} S_{1}(N+r)+2 S_{2}(N+r) \log 2-2 S_{1}(N+r) S_{2}(N+r)-S_{-2,1}(N+r)\right.\right. \\
& \left.-S_{-1,2}(N+r)-2 S_{3}(N+r)-\frac{\zeta(3)}{8}\right]+\frac{1+(-1)^{N+r}}{4}\left[S_{1}(N+r) S_{2}\left(\frac{N+r}{2}\right)+2 S_{1}\left(\frac{N+r}{2}\right) S_{2}(N+r)\right. \\
& \left.-S_{2}\left(\frac{N+r}{2}\right) \log 2+S_{3}\left(\frac{N+r}{2}\right)\right]-\frac{1-(-1)^{N+r}}{4}\left[S_{1}(N+r) S_{2}\left(\frac{N+r-1}{2}\right)\right. \\
& \left.\left.+2 S_{1}\left(\frac{N+r-1}{2}\right) S_{2}(N+r)-S_{2}\left(\frac{N+r-1}{2}\right) \log 2+S_{3}\left(\frac{N+r-1}{2}\right)\right]\right\} \\
& -\frac{2}{(N+r)^{2}}\left\{(-1)^{N+r-1}\left[S_{-1,1}(N+r)+S_{1}^{2}(N+r)+S_{2}(N+r)-2 S_{1}(N+r) \log 2\right]\right. \\
& +\frac{1+(-1)^{N+r}}{2}\left[S_{1}(N+r) S_{1}\left(\frac{N+r}{2}\right)-S_{1}\left(\frac{N+r}{2}\right) \log 2+\frac{1}{2} S_{2}\left(\frac{N+r}{2}\right)\right] \\
& \left.-\frac{1-(-1)^{N+r}}{2}\left[S_{1}(N+r) S_{1}\left(\frac{N+r-1}{2}\right)-S_{1}\left(\frac{N+r-1}{2}\right) \log 2+\frac{1}{2} S_{2}\left(\frac{N+r-1}{2}\right)-\log ^{2} 2\right]\right\}
\end{aligned}
$$

$$
\begin{aligned}
\mathbf{M}\left[z^{r} \log z \log (1+z) \log (1-z)\right](N)= & -\frac{(-1)^{N}}{N}\left[S_{-2,1}(N)+S_{-1,2}(N)-\zeta(2) S_{-1}(N)+\frac{13}{8} \zeta(3)-\frac{3}{2} \zeta(2) \log 2\right] \\
& -\frac{1}{N}\left[S_{-1}(N) S_{-2}(N)+S_{3}(N)-S_{2}(N) \log 2+S_{-2}(N) \log 2+\frac{1}{2} \zeta(2) S_{-1}(N)-\zeta(3)\right. \\
& \left.+\frac{3}{2} \zeta(2) \log 2\right]-\frac{(-1)^{N}}{N^{2}}\left[S_{-1,1}(N)+\frac{1}{2} \zeta(2)-\frac{1}{2} \log ^{2} 2\right]-\frac{1}{N^{2}}\left[\frac{1}{2} S_{2}(N)+\frac{1}{2} S_{-1}^{2}(N)\right. \\
& \left.+S_{-1}(N) \log 2-S_{1}(N) \log 2-\frac{1}{2} \zeta(2)+\frac{1}{2} \log ^{2} 2\right]
\end{aligned}
$$

$$
\begin{aligned}
\mathbf{M}\left(\frac{\log z}{1+z} \log (1+z) \log (1-z)\right)(N)= & -1)^{N}\left\{S_{1,-2,1}(N-1)+S_{1,-1,2}(N-1)+S_{-1,-1,-2}(N-1)+S_{-1,-2,-1}(N-1)\right. \\
& +S_{2,-1,1}(N-1)+S_{-2,-1,-1}(N-1)-\zeta(2) S_{1,-1}(N-1)-S_{-1,2}(N-1) \log 2 \\
& -S_{-2,1}(N-1) \log 2+S_{-1}(N-1) S_{-2}(N-1) \log 2+\frac{1}{4} \zeta(2) S_{-1}^{2}(N-1)+S_{3}(N-1) \log 2 \\
& +\left[\frac{13}{8} \zeta(3)-\frac{3}{2} \zeta(2) \log 2\right] S_{1}(N-1)-\left[\zeta(3)-\frac{3}{2} \zeta(2) \log 2\right] S_{-1}(N-1)
\end{aligned}
$$




$$
\begin{aligned}
& +\left[\frac{3}{4} \zeta(2)-\frac{1}{2} \log ^{2} 2\right] S_{2}(N-1)-\frac{1}{2}\left[\zeta(2)-\log ^{2} 2\right] S_{-2}(N-1)-2 \operatorname{Li}_{4}\left(\frac{1}{2}\right)+\frac{4}{5} \zeta^{2}(2) \\
& \left.-\frac{21}{8} \zeta(3) \log 2+\frac{5}{4} \zeta(2) \log ^{2} 2-\frac{1}{12} \log ^{4} 2\right\} \text {, } \\
& \mathbf{M}\left[z^{r} \operatorname{Li}_{2}(z)\right](N)=\frac{1}{N+r}\left[\zeta(2)-\frac{S_{1}(N+r)}{N+r}\right], \\
& \mathbf{M}\left[\left(\frac{1}{1-z}\right)_{+} \operatorname{Li}_{2}(z)\right](N)=-\zeta(2) S_{1}(N-1)+S_{2,1}(N-1)-2 \zeta(3), \\
& \mathbf{M}\left(\frac{\operatorname{Li}_{2}(z)}{1+z}\right)(N)=(-1)^{N-1}\left[\zeta(2) S_{-1}(N-1)-S_{-2,1}(N-1)-\frac{5}{8} \zeta(3)+\zeta(2) \log 2\right], \\
& \mathbf{M}\left(\frac{\mathrm{Li}_{2}(z)}{1-z} \log z\right)(N)=2 \zeta(2) S_{2}(N-1)-\frac{1}{2} S_{2}^{2}(N-1)-\frac{1}{2} S_{4}(N-1)-2 S_{3,1}(N-1)-\frac{3}{10} \zeta^{2}(2), \\
& \mathbf{M}\left[z^{r} \operatorname{Li}_{2}(z) \log z\right](N)=\frac{1}{(N+r)^{2}}\left[-2 \zeta(2)+\frac{2 S_{1}(N+r)}{N+r}+S_{2}(N+r)\right], \\
& \mathbf{M}\left[z^{r} \operatorname{Li}_{2}(z) \log (1-z)\right](N)=\frac{1}{N+r}\left\{-2 \zeta(3)-\zeta(2) S_{1}(N+r)+\frac{1}{N+r}\left[S_{1}^{2}(N+r)+S_{2}(N+r)\right]+S_{2,1}(N+r)\right\}, \\
& \mathbf{M}\left[\left(\frac{\log (1-z)}{1-z}\right)_{+} \operatorname{Li}_{2}(z)\right](N)=-2 S_{2,1,1}(N-1)-S_{1,2,1}(N-1)+\frac{1}{2} \zeta(2) S_{1}^{2}(N-1)+\frac{1}{2} \zeta(2) S_{2}(N-1)+2 \zeta(3) S_{1}(N-1) \\
& +\frac{6}{5} \zeta^{2}(2), \\
& \mathbf{M}\left[z^{r} \operatorname{Li}_{2}(-z)\right](N)=\frac{1}{N+r}\left\{-\frac{\zeta(2)}{2}+\frac{1+(-1)^{N+r-1}}{N+r} \log (2)+\frac{(-1)^{N+r-1}}{N+r}\left[-S_{1}(N+r)+\frac{1+(-1)^{N+r-1}}{2} S_{1}\left(\frac{N+r-1}{2}\right)\right.\right. \\
& \left.\left.+\frac{1-(-1)^{N+r+1}}{2} S_{1}\left(\frac{N+r}{2}\right)\right]\right\} \\
& \mathbf{M}\left(\frac{\operatorname{Li}_{2}(-z)}{1+z}\right)(N)=(-1)^{N-1}\left\{-\left[S_{2}(N-1)+\frac{\zeta(2)}{2}\right]\left[\frac{1+(-1)^{N-1}}{2} S_{1}\left(\frac{N-1}{2}\right)+\frac{1-(-1)^{N-1}}{2} S_{1}\left(\frac{N-2}{2}\right)-S_{1}(N-1)\right]\right. \\
& +\left[\frac{1+(-1)^{N-1}}{4} S_{2}\left(\frac{N-1}{2}\right)+\frac{1-(-1)^{N-1}}{4} S_{2}\left(\frac{N-2}{2}\right)-2 S_{2}(N-1)\right] \log 2-\frac{1+(-1)^{N-1}}{8} S_{3}\left(\frac{N-1}{2}\right) \\
& \left.-\frac{1-(-1)^{N-1}}{8} S_{3}\left(\frac{N-2}{2}\right)+S_{3}(N-1)+S_{-1,2}(N-1)-\frac{\zeta(2)}{2} \log 2+\frac{\zeta(3)}{4}\right\} \text {, } \\
& \mathbf{M}\left[z^{r} \operatorname{Li}_{2}(-z) \log z\right](N)=\frac{(-1)^{N+r-1}}{(N+r)^{2}}\left[\frac{2 S_{1}(N+r)}{N+r}+S_{2}(N+r)\right]-\frac{1+(-1)^{N+r-1}}{2(N+r)^{2}}\left[\frac{2}{N+r} S_{1}\left(\frac{N+r-1}{2}\right)\right. \\
& \left.+\frac{1}{2} S_{2}\left(\frac{N+r-1}{2}\right)+\frac{4 \log 2}{N+r}\right]+\frac{1-(-1)^{N+r-1}}{2(N+r)^{2}}\left[\frac{2}{N+r} S_{1}\left(\frac{N+r}{2}\right)+\frac{1}{2} S_{2}\left(\frac{N+r}{2}\right)+\zeta(2)\right],
\end{aligned}
$$




$$
\begin{aligned}
& \mathbf{M}\left(\frac{\mathrm{Li}_{2}(-z)}{1-z} \log z\right)(N)=-2 S_{-3,-1}(N-1)-\frac{1}{2} S_{-2}^{2}(N-1)-\frac{1}{2} S_{4}(N-1)+2 S_{3}(N-1) \log 2-2 S_{-3}(N-1) \log 2 \\
& -\frac{1}{2} \zeta(2) S_{2}(N-1)-\frac{1}{2} \zeta(2) S_{-2}(N-1)-4 \mathrm{Li}_{4}\left(\frac{1}{2}\right)+\frac{71}{40} \zeta^{2}(2)-\frac{7}{2} \zeta(3) \log 2+\zeta(2) \log ^{2} 2-\frac{1}{6} \log ^{4} 2, \\
& \mathbf{M}\left(\frac{\operatorname{Li}_{2}(-z)}{1+z} \log z\right)(N)=(-1)^{N-1}\left[2 S_{3,-1}(N-1)+S_{2,-2}(N-1)-2 S_{-3}(N-1) \log 2+2 S_{3}(N-1) \log 2+\frac{1}{2} \zeta(2) S_{2}(N-1)\right. \\
& \left.+\frac{1}{2} \zeta(2) S_{-2}(N-1)-4 \operatorname{Li}_{4}\left(\frac{1}{2}\right)+\frac{13}{8} \zeta^{2}(2)-\frac{7}{2} \zeta(3) \log 2+\zeta(2) \log ^{2} 2-\frac{1}{6} \log ^{4} 2\right] \\
& \mathbf{M}\left[z^{r} \operatorname{Li}_{2}(-z) \log (1-z)\right](N)=\frac{(-1)^{N+r}}{(N+r)^{2}}\left[S_{-1,1}(N+r)+\frac{1}{2} \zeta(2)-\frac{1}{2} \log ^{2} 2\right]+\frac{1}{(N+r)^{2}}\left[\frac{1}{2} S_{-1}^{2}(N+r)+\frac{1}{2} S_{2}(N+r)\right. \\
& \left.-S_{1}(N+r) \log 2+S_{-1}(N+r) \log 2-\frac{1}{2} \zeta(2)+\frac{1}{2} \log ^{2} 2\right]+\frac{1}{N+r}\left[S_{-2,-1}(N+r)\right. \\
& \left.+\frac{1}{2} \zeta(2) S_{1}(N+r)-S_{2}(N+r) \log 2+S_{-2}(N+r) \log 2+\frac{5}{8} \zeta(3)\right] \\
& \mathbf{M}\left[z^{r} \operatorname{Li}_{2}(-z) \log (1+z)\right](N)=\frac{(-1)^{N+r}}{N+r}\left\{[ S _ { 2 } ( N + r ) + \frac { \zeta ( 2 ) } { 2 } ] \left[\frac{1+(-1)^{N+r}}{2} S_{1}\left(\frac{N+r}{2}\right)+\frac{1-(-1)^{N+r}}{2} S_{1}\left(\frac{N+r-1}{2}\right)\right.\right. \\
& \left.-S_{1}(N+r)\right]-\left[\frac{1+(-1)^{N+r}}{4} S_{2}\left(\frac{N+r}{2}\right)+\frac{1-(-1)^{N+r}}{4} S_{2}\left(\frac{N+r-1}{2}\right)-2 S_{2}(N+r)\right] \log 2 \\
& +\frac{1+(-1)^{N+r}}{8} S_{3}\left(\frac{N+r}{2}\right)+\frac{1-(-1)^{N+r}}{8} S_{3}\left(\frac{N+r-1}{2}\right)-S_{3}(N+r)-S_{-1,2}(N+r) \\
& \left.+\frac{1-(-1)^{N+r}}{2} \zeta(2) \log 2-\frac{\zeta(3)}{4}\right\}+\frac{2(-1)^{N+r}}{(N+r)^{2}}\left\{-S_{-1,1}(N+r)-S_{1}^{2}(N+r)-S_{2}(N+r)\right. \\
& +2 S_{1}(N+r) \log 2+\frac{1+(-1)^{N+r}}{2}\left[S_{1}(N+r) S_{1}\left(\frac{N+r}{2}\right)-S_{1}\left(\frac{N+r}{2}\right) \log 2+\frac{1}{2} S_{2}\left(\frac{N+r}{2}\right)\right] \\
& \left.+\frac{1-(-1)^{N+r}}{2}\left[S_{1}(N+r) S_{1}\left(\frac{N+r-1}{2}\right)-S_{1}\left(\frac{N+r-1}{2}\right) \log 2+\frac{1}{2} S_{2}\left(\frac{N+r-1}{2}\right)-\log ^{2} 2\right]\right\},
\end{aligned}
$$

$$
\begin{aligned}
\mathbf{M}\left(\frac{\operatorname{Li}_{2}(-z)}{1+z} \log (1-z)\right)(N)= & -1)^{N-1}\left\{S_{2,-1,1}(N-1)+S_{-2,-1,-1}(N-1)+S_{-1,-2,-1}(N-1)-S_{-2,1}(N-1) \log 2\right. \\
& -S_{-1,2}(N-1) \log 2+\frac{1}{2} \zeta(2) S_{-1,1}(N-1)+S_{-1}(N-1) S_{-2}(N-1) \log 2+S_{3}(N-1) \log 2 \\
& +\frac{1}{2}\left[\zeta(2)-\log ^{2}(2)\right]\left[S_{2}(N-1)-S_{-2}(N-1)\right]+\frac{5}{8} \zeta(3) S_{-1}(N-1)-4 \operatorname{Li}_{4}\left(\frac{1}{2}\right) \\
& \left.+\frac{3}{2} \zeta^{2}(2)-\frac{21}{8} \zeta(3) \log 2+\frac{3}{4} \zeta(2) \log ^{2} 2-\frac{1}{6} \log ^{4} 2\right\}
\end{aligned}
$$




$$
\begin{aligned}
& \mathbf{M}\left(\frac{\operatorname{Li}_{2}(-z)}{1+z} \log (1+z)\right)(N)=(-1)^{N-1}\left\{S_{1,2,-1}(N-1)+2 S_{2,1,-1}(N-1)+\left[S_{2,1}(N-1)-S_{1,-2}(N-1)-2 S_{2,-1}(N-1)\right.\right. \\
& \left.+S_{1}(N-1) S_{2}(N-1)+S_{3}(N-1)-\frac{1}{2} \zeta(2) S_{-1}(N-1)\right] \log 2+\frac{1}{2} \zeta(2) S_{1,-1}(N-1) \\
& -\left[S_{2}(N-1)-S_{-2}(N-1)\right] \log ^{2} 2-\left[\frac{1}{4} \zeta(3)-\frac{1}{2} \zeta(2) \log 2\right] S_{1}(N-1)-3 \operatorname{Li}_{4}\left(\frac{1}{2}\right) \\
& \left.+\frac{6}{5} \zeta^{2}(2)-\frac{21}{8} \zeta(3) \log 2+\frac{1}{2} \zeta(2) \log ^{2} 2-\frac{1}{8} \log ^{4} 2\right\}, \\
& \mathbf{M}\left[\operatorname{Li}_{2}(1-z)\right](N)=-\frac{1}{N}\left[S_{2}(N)-\zeta(2)\right] \\
& \mathbf{M}\left[\operatorname{Li}_{2}(1-z) \log z\right](N)=\frac{1}{N^{2}}\left[S_{2}(N)-\zeta(2)\right]+\frac{2}{N}\left[S_{3}(N)-\zeta(3)\right], \\
& \mathbf{M}\left[\operatorname{Li}_{2}(1-z) \log (1-z)\right](N)=\frac{2}{N}\left[S_{1}(N) S_{2}(N)-\zeta(2) S_{1}(N)+S_{3}(N)-\frac{1}{2} S_{2,1}(N)\right], \\
& \mathbf{M}\left(\frac{\operatorname{Li}_{2}(1-z)}{1-z}\right)(N)=S_{1}(N-1) S_{2}(N-1)-\zeta(2) S_{1}(N-1)+S_{3}(N-1)-S_{2,1}(N-1)+\zeta(3), \\
& \mathbf{M}\left(\operatorname{Li}_{2}(1-z) \frac{\log (1-z)}{1-z}\right)(N)=-2 S_{1,1,2}(N-1)-S_{1,2,1}(N-1)+\zeta(2) S_{1}^{2}(N-1)+\zeta(2) S_{2}(N-1)-\frac{2}{5} \zeta^{2}(2), \\
& \mathbf{M}\left(\operatorname{Li}_{2}(1-z) \frac{\log z}{1-z}\right)(N)=-2 S_{1}(N-1) S_{3}(N-1)+2 \zeta(3) S_{1}(N-1)-\frac{1}{2} S_{2}^{2}(N-1)+\zeta(2) S_{2}(N-1)-\frac{5}{2} S_{4}(N-1) \\
& +2 S_{3,1}(N-1)-\frac{1}{2} \zeta(2)^{2} \\
& \mathbf{M}\left(\frac{\operatorname{Li}_{2}(1-z) \log z}{1+z}\right)(N)=(-1)^{N-1}\left[S_{-2,2}(N-1)+2 S_{-1,3}(N-1)-\zeta(2) S_{-2}(N-1)-2 \zeta(3) S_{-1}(N-1)+4 \operatorname{Li}_{4}\left(\frac{1}{2}\right)\right. \\
& \left.-\frac{33}{40} \zeta^{2}(2)-\zeta(2) \log ^{2} 2+\frac{1}{6} \log ^{4} 2\right] \\
& \mathbf{M}[\widetilde{\Phi}(z)](N)=\frac{1}{N^{3}}+2 \frac{(-1)^{N}}{N}\left[S_{2}(N)-\zeta(2)\right]-\frac{1+(-1)^{N}}{2 N}\left[S_{2}\left(\frac{N}{2}\right)-\zeta(2)\right]+\frac{1-(-1)^{N}}{2 N}\left[S_{2}\left(\frac{N-1}{2}\right)-\zeta(2)\right] \\
& \mathbf{M}\left(\frac{\widetilde{\Phi}(z)}{1+z}\right)(N)=(-1)^{N-1}\left\{-\zeta(2) S_{1}(N-1)-2 S_{1}(N-1)\left[\frac{1+(-1)^{N-1}}{4} S_{2}\left(\frac{N-1}{2}\right)+\frac{1-(-1)^{N-1}}{4} S_{2}\left(\frac{N-2}{2}\right)-S_{2}(N-1)\right]\right. \\
& \left.-\frac{1+(-1)^{N-1}}{8} S_{3}\left(\frac{N-1}{2}\right)-\frac{1-(-1)^{N-1}}{8} S_{3}\left(\frac{N-2}{2}\right)+S_{3}(N-1)+2 S_{-2,1}(N-1)+\frac{\zeta(3)}{2}\right\}
\end{aligned}
$$




$$
\begin{aligned}
& \mathbf{M}\left(\widetilde{\Phi}(z) \frac{\log (1-z)}{1+z}\right)(N)=(-1)^{N-1}\left\{2 S_{1,-2,1}(N-1)+2 S_{1,-1,2}(N-1)+2 S_{-1,-1,-2}(N-1)-S_{-2,2}(N-1)-S_{-1,3}(N-1)\right. \\
& -S_{-3,1}(N-1)-2 \zeta(2) S_{1,-1}(N-1)+\frac{1}{2} \zeta(2) S_{-1}^{2}(N-1)+\frac{1}{2} \zeta(2) S_{2}(N-1)+\zeta(2) S_{-2}(N-1) \\
& +\left[\frac{13}{4} \zeta(3)-3 \zeta(2) \log 2\right] S_{1}(N-1)-\left[\frac{9}{4} \zeta(3)-3 \zeta(2) \log 2\right] S_{-1}(N-1)+2 \operatorname{Li}_{4}\left(\frac{1}{2}\right) \\
& \left.-\frac{7}{10} \zeta^{2}(2)+\zeta(2) \log ^{2} 2+\frac{1}{12} \log ^{4} 2\right\} \\
& \mathbf{M}\left[z^{r} \operatorname{Li}_{3}(z)\right](N)=\frac{1}{N+r}\left[\zeta(3)-\frac{\zeta(2)}{N+r}+\frac{S_{1}(N+r)}{(N+r)^{2}}\right], \\
& \mathbf{M}\left[\left(\frac{1}{1-z}\right)+\operatorname{Li}_{3}(z)\right](N)=-\zeta(3) S_{1}(N-1)+\zeta(2) S_{2}(N-1)-S_{3,1}(N-1)-\frac{1}{2} \zeta(2)^{2}, \\
& \mathbf{M}\left[z^{r} \operatorname{Li}_{3}(-z)\right](N)=(-1)^{N+r-1} \frac{S_{1}(N+r)}{(N+r)^{3}}-\frac{1+(-1)^{N+r-1}}{2(N+r)^{3}}\left[S_{1}\left(\frac{N+r-1}{2}\right)+2 \log 2\right]+\frac{1-(-1)^{N+r-1}}{2(N+r)^{3}} S_{1}\left(\frac{N+r}{2}\right) \\
& +\frac{\zeta(2)}{2(N+r)^{2}}-\frac{3 \zeta(3)}{4(N+r)} \\
& \mathbf{M}\left[\left(\frac{1}{1-z}\right)_{+} \operatorname{Li}_{3}(-z)\right](N)=-S_{-3,-1}(N-1)+\left[S_{3}(N-1)-S_{-3}(N-1)\right] \log 2-\frac{1}{2} \zeta(2) S_{2}(N-1)+\frac{3}{4} \zeta(3) S_{1}(N-1) \\
& -2 \operatorname{Li}_{4}\left(\frac{1}{2}\right)+\frac{11}{10} \zeta^{2}(2)-\frac{7}{4} \zeta(3) \log 2+\frac{1}{2} \zeta(2) \log ^{2} 2-\frac{1}{12} \log ^{4} 2 \\
& \mathbf{M}\left(\frac{\operatorname{Li}_{3}(-z)}{1+z}\right)(N)=(-1)^{N-1}\left\{S_{3,-1}(N-1)+\left[S_{3}(N-1)-S_{-3}(N-1)\right] \log 2+\frac{1}{2} \zeta(2) S_{-2}(N-1)-\frac{3}{4} \zeta(3) S_{-1}(N-1)\right. \\
& \left.+\frac{1}{8} \zeta^{2}(2)-\frac{3}{4} \zeta(3) \log 2\right\} \\
& \mathbf{M}\left[\operatorname{Li}_{3}(1-z)\right](N)=\frac{1}{N}\left[S_{1}(N) S_{2}(N)-\zeta(2) S_{1}(N)+S_{3}(N)-S_{2,1}(N)+\zeta(3)\right], \\
& \mathbf{M}\left(\frac{\operatorname{Li}_{3}(1-z)}{1-z}\right)(N)=-S_{1,1,2}(N-1)+\frac{1}{2} \zeta(2) S_{1}^{2}(N-1)+\frac{1}{2} \zeta(2) S_{2}(N-1)-\zeta(3) S_{1}(N-1)+\frac{2}{5} \zeta^{2}(2) \\
& \mathbf{M}\left(\frac{\operatorname{Li}_{3}(1-z)}{1+z}\right)(N)=(-1)^{N-1}\left[S_{-1,1,2}(N-1)-\zeta(2) S_{-1,1}(N-1)+\zeta(3) S_{-1}(N-1)+\operatorname{Li}_{4}\left(\frac{1}{2}\right)-\frac{9}{20} \zeta^{2}(2)+\frac{7}{8} \zeta(3) \log 2\right. \\
& \left.+\frac{1}{2} \zeta(2) \log ^{2} 2+\frac{1}{24} \log ^{4} 2\right],
\end{aligned}
$$

$$
\begin{aligned}
\mathbf{M}\left[\operatorname{Li}_{3}\left(\frac{1-z}{1+z}\right)-\mathrm{Li}_{3}\left(-\frac{1-z}{1+z}\right)\right](N)= & \frac{(-1)^{N}}{N}\left[-S_{-1,2}(N)-S_{-2,1}(N)+S_{1}(N) S_{-2}(N)+S_{-3}(N)+\zeta(2) S_{-1}(N)+\frac{1}{2} \zeta(2) S_{1}(N)\right. \\
& \left.-\frac{7}{8} \zeta(3)+\frac{3}{2} \zeta(2) \log 2\right]+\frac{1}{N}\left[-S_{-1,-2}(N)-S_{2,1}(N)+S_{1}(N) S_{2}(N)+S_{3}(N)\right. \\
& \left.-\frac{1}{2} \zeta(2) S_{-1}(N)-\zeta(2) S_{1}(N)+\frac{21}{8} \zeta(3)-\frac{3}{2} \zeta(2) \log 2\right]
\end{aligned}
$$




$$
\begin{aligned}
& \mathbf{M}\left\{\frac{1}{1+z}\left[\operatorname{Li}_{3}\left(\frac{1-z}{1+z}\right)-\operatorname{Li}_{3}\left(-\frac{1-z}{1+z}\right)\right]\right\}(N)=(-1)^{N-1}\left\{S_{1,1,-2}(N-1)-S_{1,-1,2}(N-1)+S_{-1,1,2}(N-1)-S_{-1,-1,-2}(N-1)\right. \\
& +2 \zeta(2) S_{1,-1}(N-1)+\frac{1}{4} \zeta(2) S_{1}^{2}(N-1)-\frac{1}{4} \zeta(2) S_{-1}^{2}(N-1)-\zeta(2) S_{1}(N-1) \\
& \times S_{-1}(N-1)-\zeta(2) S_{-2}(N-1)-\left[\frac{7}{8} \zeta(3)-\frac{3}{2} \zeta(2) \log 2\right] S_{1}(N-1)+\left[\frac{21}{8} \zeta(3)\right. \\
& \left.\left.-\frac{3}{2} \zeta(2) \log 2\right] S_{-1}(N-1)-2 \operatorname{Li}_{4}\left(\frac{1}{2}\right)+\frac{19}{40} \zeta^{2}(2)+\frac{1}{2} \zeta(2) \log ^{2} 2-\frac{1}{12} \log ^{4} 2\right\}, \\
& \mathbf{M}\left[z^{r} \mathrm{~S}_{1,2}(z)\right](N)=\frac{\zeta(3)}{N+r}-\frac{S_{1}^{2}(N+r)+S_{2}(N+r)}{2(N+r)^{2}} \\
& \mathbf{M}\left[\left(\frac{1}{1-z}\right)_{+} \mathrm{S}_{1,2}(z)\right](N)=S_{2,1,1}(N-1)-\zeta(3) S_{1}(N-1)-\frac{6}{5} \zeta^{2}(2), \\
& \mathbf{M}\left(\frac{\mathrm{S}_{1,2}(z)}{1+z}\right)(N)=(-1)^{N}\left[S_{-2,1,1}(N-1)-\zeta(3) S_{-1}(N-1)+\operatorname{Li}_{4}\left(\frac{1}{2}\right)-\frac{1}{8} \zeta^{2}(2)-\frac{1}{8} \zeta(3) \log 2-\frac{1}{4} \zeta(2) \log ^{2} 2+\frac{1}{24} \log ^{4} 2\right] \\
& +\frac{1-(-1)^{N}}{2 N^{2}}\left[S_{1}(N) S_{1}\left(\frac{N-1}{2}\right)-S_{1}\left(\frac{N-1}{2}\right) \log 2+\frac{1}{2} S_{2}\left(\frac{N-1}{2}\right)-\log ^{2} 2\right]+\frac{\zeta(3)}{8 N}, \\
& \mathbf{M}\left(\frac{\mathrm{S}_{1,2}(-z)}{1+z}\right)(N)=(-1)^{N}\left\{S_{2,1,-1}(N-1)+\left[S_{2,1}(N-1)-S_{2,-1}(N-1)\right] \log 2-\frac{1}{2}\left[S_{2}(N-1)-S_{-2}(N-1)\right] \log ^{2} 2\right. \\
& \left.-\frac{1}{8} \zeta(3) S_{-1}(N-1)-3 \operatorname{Li}_{4}\left(\frac{1}{2}\right)+\frac{6}{5} \zeta^{2}(2)-\frac{11}{4} \zeta(3) \log 2+\frac{3}{4} \zeta(2) \log ^{2} 2-\frac{1}{8} \log ^{4} 2\right\} \\
& \mathbf{M}\left[\mathrm{S}_{1,2}(1-z)\right](N)=-\frac{1}{N}\left[S_{3}(N)-\zeta(3)\right] \\
& \mathbf{M}\left(\frac{\mathrm{S}_{1,2}(1-z)}{1-z}\right)(N)=S_{1}(N-1) S_{3}(N-1)-\zeta(3) S_{1}(N-1)+S_{4}(N-1)-S_{3,1}(N-1)+\frac{\zeta(2)^{2}}{10} \\
& \mathbf{M}\left(\frac{\mathrm{S}_{1,2}(1-z)}{1+z}\right)(N)=(-1)^{N}\left[S_{-1,3}(N-1)-\zeta(3) S_{-1}(N-1)+\frac{19}{40} \zeta^{2}(2)-\frac{7}{4} \zeta(3) \log 2\right] .
\end{aligned}
$$

[1] For older approaches see Refs. [46,48] in J. Blümlein, Surv. High Energy Phys. 7, 181 (1994); A. Devoto, D. W. Duke, J. F. Owens, and R. G. Roberts, Phys. Rev. D 27, 508 (1983); M. Virchaux and A. Ouraou, DphPE 87-15; M. Virchaux, Thèse, Université Paris-7, 1988; A. Ouraou, Thèse, Université Paris11, 1988; M. Botje, QCDNUM15: A fast QCD evolution Program; C. Pascaud and F. Zomer, H1 Note: H1-11/94-404;
CTEQ Collaboration, H. Lai et al., Phys. Rev. D 55, 1280 (1997), and references therein.

[2] M. Diemoz, F. Ferroni, E. Longo, and G. Martinelli, Z. Phys. C 39, 21 (1988); M. Glück, E. Reya, and A. Vogt, ibid. 48, 471 (1990); J. Blümlein, A. Kryukov, W.L. van Neerven, and A. Vogt (in preparation).

[3] H. Mellin, Acta Math. 25, 139 (1902). 
[4] H. Triebel, Höhere Analysis (DVW, Berlin, 1972).

[5] J. Blümlein, V. Ravindran, and W. L. van Neerven, Acta Phys. Pol. B 29, 2581 (1998); DESY 98-144.

[6] J. Blümlein and A. Vogt, Phys. Lett. B 370, 149 (1996); Acta Phys. Pol. B 27, 1309 (1996); Phys. Lett. B 386, 350 (1996); J. Blümlein, S. Riemersma, and A. Vogt, Nucl. Phys. B (Proc. Suppl.) 51C, 50 (1996); Eur. Phys. J. C 1, 255 (1998); J. Blümlein and A. Vogt, Phys. Rev. D 57, 1 (1998); J. Blümlein, V. Ravindran, W. L. van Neerven, and A. Vogt, DESY 98067, hep-ph/9806368.

[7] J. Blümlein and A. Vogt, Phys. Rev. D 58, 014020 (1998).

[8] R. K. Ellis, Z. Kunszt, and E. Levin, Nucl. Phys. B420, 517 (1994); B433, 498(E) (1995).

[9] C. Berger, D. Graudenz, M. Hampel, and A. Vogt, Z. Phys. C 70, 77 (1996); For a further application see D. Kosower, Nucl. Phys. B520, 263 (1998).

[10] K. Yoshida, Functional Analysis (Springer, Berlin, 1978), Chap. XI.

[11] L. Schwartz, Théorie des Distributions (Hermann and Cie, Paris, 1951), Vols. I and II; V. S. Vladimirov, Gleichungen der Mathematischen Physik (DVW, Berlin, 1972).

[12] E. Nielsen, Nova Acta Leopold. XC, 121 (1909).

[13] S. Kölbig, Siam J. Math. Anal. 17, 1232 (1986).

[14] L. Lewin, Dilogarithms and Associated Functions (Macdonald, London, 1958); Polylogarithms and Associated Functions (North Holland, New York, 1981).

[15] L. Euler, Novi Comm. Acad. Sci Petropolitanae 1, 140 (1775).

[16] D. Zagier, in Proceedings of the First European Congress on Mathematics (Birkhäuser, Boston, 1994), Vol. II, pp. 497.

[17] C. Kassel, Quantum Groups (Springer, Berlin, 1995).

[18] J. M. Borwein and R. Girgensohn, Electron. J. Combinatorics 3, R23 (1996) (Appendix by D. J. Broadhurst).

[19] D. J. Broadhurst, OUT-4102-62, hep-th/9604128.

[20] J. Blümlein (in preparation).

[21] E. B. Zijlstra and W. L. van Neerven, Phys. Lett. B 273, 476 (1991); Nucl. Phys. B383, 525 (1992); E. B. Zijlstra, Ph.D. thesis, Leiden University, 1993.

[22] W. L. van Neerven and E. B. Zijlstra, Phys. Lett. B 272, 127 (1991).

[23] E. B. Zijlstra and W. L. van Neerven, Phys. Lett. B 297, 377 (1992); Nucl. Phys. B417, 61 (1994); B426, 245(E) (1994).

[24] P. J. Rijken and W. L. van Neerven, Phys. Lett. B 386, 422 (1996); Nucl. Phys. B487, 233 (1997); Phys. Lett. B 392, 207 (1997); Nucl. Phys. B523, 245 (1998).

[25] A. N. Schellekens and W. L. van Neerven, Phys. Rev. D 22, 1623 (1980); 21, 2619 (1980); B. Humpert and W. L. van Neerven, Phys. Lett. 85B, 293 (1979); T. Matsuura and W. L. van Neerven, Z. Phys. C 38, 623 (1988); T. Matsuura, S. C. van der Marck, and W. L. van Neerven, Phys. Lett. B 211, 171 (1988); Nucl. Phys. B319, 570 (1990); T. Matsuura, R. Hamberg, and W. L. van Neerven, ibid. B345, 331 (1990); R. Hamberg, W. L. van Neerven, and T. Matsuura, ibid. B395, 343 (1991); W. L. van Neerven and E. B. Zijlstra, ibid. B382, 11 (1992); W. L. van Neerven, Int. J. Mod. Phys. A 10, 2921 (1995); P. J. Rijken and W. L. van Neerven, Phys. Rev. D 51, 44 (1995); 52, 149 (1995).

[26] D. Gross and F. Wilczek, Phys. Rev. D 8, 3633 (1973); 9, 980 (1974); H. Georgi and D. Politzer, ibid. 9, 416 (1974).

[27] L. N. Lipatov, Yad. Fiz. 20, 181 (1977) [Sov. J. Nucl. Phys.
20, 94 (1975)]; G. Altarelli and G. Parisi, Nucl. Phys. B126, 298 (1977); K. Kim and L. Schilcher, Phys. Rev. D 17, 2800 (1978); Yu. Dokshitser, Zh. Éksp. Teor. Fiz. 73, 1216 (1977) [Sov. Phys. JETP 46, 641 (1977)]; H. Ito, Prog. Theor. Phys. 54, 555 (1975); K. Sasaki, ibid. 54, 1816 (1975); M. Ahmed and G. G. Ross, Phys. Lett. 56B, 385 (1975); Nucl. Phys. B111, 298 (1976).

[28] G. Floratos, P. Lacaze, and C. Kounnas, Nucl. Phys. B192, 417 (1981); A. Gonzalez-Arroyo and C. Lopez, ibid. B166, 429 (1980); G. Floratos, P. Lacaze, and C. Kounnas, Phys. Lett. 98B, 285 (1981); W. Furmanski and R. Petronzio, ibid. 97B, 437 (1980); R. K. Ellis and W. Vogelsang, FERMILABPUB-96-044-T, hep-ph/9602356; R. Mertig and W. L. van Neerven, Z. Phys. C 70, 637 (1996); W. Vogelsang, Phys. Rev. D 54, 2023 (1996); Nucl. Phys. B475, 47 (1996).

[29] S. Larin, T. van Ritbergen, and J. Vermaseren, Nucl. Phys. B427, 41 (1994); S. Larin, P. Nogueira, T. van Ritbergen, and J. Vermaseren, ibid. B492, 338 (1997).

[30] W. Gröbner and N. Hofreiter, Integraltafeln (Springer, Wien, 1973), Vol. II.

[31] R. Barbieri, J. A. Mignaco, and E. Remiddi, Nuovo Cimento A 11, 824 (1972); J. A. Mignaco and E. Remiddi, ibid. 60, 519 (1969); M. J. Levine, E. Remiddi, and R. Roskies, Phys. Rev. D 20, 2068 (1979).

[32] D. H. Bailey, J. Borwein, and R. Girgensohn, Experiment. Math. 3, 17 (1994).

[33] D. Borwein, J. M. Borwein, and R. Girgensohn, Proc. Edinburgh Math. Soc. 38, 277 (1995).

[34] D. Broadhurst and D. Kreimer, Phys. Lett. B 393, 403 (1997).

[35] J. Vermaseren, hep-ph/9806280, FTUAM 98-7, NIKHEF-9814.

[36] Ph. Flajolet and B. Salvy, Exp. Mathematics 7, 15 (1998); J. M. Borwein, D.M. Bradley, D.J. Broadhurst, and P. Lisonek, CECM Research Report 98-106.

[37] A. González-Arroyo, C. López, and F. J. Ynduráin, Nucl. Phys. B153, 161 (1979); F. J. Ynduráin, The Theory of Quark and Gluon Interactions (Springer, Berlin, 1993).

[38] A. Zee, F. Wilczek, and S. B. Treiman, Phys. Rev. D 10, 2881 (1974).

[39] D. W. Duke, J. D. Kimel, and G. A. Sowell, Phys. Rev. D 25, 71 (1982); S. N. Coulson and R. E. Ecclestone, Phys. Lett. 115B, 415 (1982); Nucl. Phys. B211, 317 (1983); A. Devoto, D. W. Duke, J. D. Kimel, and G. A. Sowell, Phys. Rev. D 30, 541 (1984); J. L. Miramontes, J. Sanchez Guillén, and E. Zas, ibid. 35, 863 (1987); J. Sanchez Guillén et al., Nucl. Phys. B353, 337 (1991).

[40] D. I. Kazakov et al., Phys. Rev. Lett. 65, 1535 (1990); 65, 1921(E) (1990); D. I. Kazakov and A. V. Kotikov, Nucl. Phys. B307, 721 (1988); B345, 299(E) (1990).

[41] S. Larin and J. Vermaseren, Z. Phys. C 57, 93 (1991).

[42] D. I. Kazakov and A. V. Kotikov, Phys. Lett. B 291, 171 (1992).

[43] J. Blümlein, DESY 98-149.

[44] J. Vermaseren, Acta Phys. Pol. B 29, 2599 (1998).

[45] H. Poincaré, Acta Math. 4, 201 (1884); J. A. LappoDanilevsky, Mémoirs sur la Théorie des Systèmes Différentielles Linéaires (Chelsea Publishing Company, New York, 1953). These representations were very often used in calculating Feynamn diagrams. Later these representations were also 
called Drinfeld integrals [16] in a different context; see also [17] and references therein and Tu Quoc Thang Le and J. Murakami, Top. Appl. 62, 193 (1995).

[46] R. Gastmans and W. Troost, Simon Stevin 55, 205 (1981).

[47] A. Devoto and D. W. Duke, Riv. Nuovo Cimento 7, 1 (1984). [48] J. Blümlein (unpublished).

[49] R. L. Graham, D.E. Knuth, and O. Patashnik, Concrete Mathematics (Addison-Wesley, Reading/MA, 1994).

[50] N. Nielsen, Handbuch der Theorie der Gammafunktion (Teubner, Leipzig, 1906), pp. 44: reprinted by Chelsea Publishing Company, Bronx, NY 1965.

[51] N. Nielsen, Ann. Math. 9, 219 (1904); N. Nielsen, Ref. [12]; P. S. Bruckman, Fibonacci Quart. 20, 186 (1982); Y. Matsuoka, Tokyo J. Math. 19, 85 (1984); T. M. Apostol and T. H. Vu, J. Number Theory 19, 85 (1984).

[52] M. Dehn, Math. Ann. 55, 465 (1902); W. F. Kagan, ibid. 57, 421 (1903).

[53] D. Hilbert, Nachr. der Kgl. Ges. d. Wiss. zu Göttingen, math.phys. Klasse 3, 253 (1900).
[54] R. Sita Ramachandra Rao and M. V. Subbarao, Pac. J. Math. 113, 471 (1984).

[55] M. E. Hoffman and C. Moen, J. Number Theory 60, 329 (1996); M. E. Hoffman, Pac. J. Math. 152, 275 (1992); J. Algebra P 194, 477 (1997).

[56] The Catholic Encyclopedia, www.knight.org/advent, 1997; http://www.-history.mcs.st.andrews.ac. uk/history/Mathematicians/ Faa_di-_Bruno.html

[57] F. Faà di Bruno, Einleitung in die Theorie dier Binären Formen, dt. Bearbeitung von Th. Walter (Teubner, Leipzig, 1881).

[58] B.C. Berndt, Ramanujan's Notebooks, Part I (Springer, Berlin, 1985).

[59] J. Binet, J. de l'école Polytechn., cahier 27, 123 (1839).

[60] W. Scheibner, Leipziger Berichte 14, 75 (1862).

[61] L. Comtet, Advanced Combinatorics (Reidel, Dordrecht, 1974); V. Adamcik, J. Comput. Appl. Math. 79, 119 (1997).

[62] C. Markett, J. Number Theory 48, 113 (1994).

[63] For a uniqueness theorem, see E. Carlson, thesis, Univ. Uppsala, 1914; E. C. Titchmarsh, Theory of Functions (Oxford University Press, Oxford, 1939), Chap. 9.5. 